\title{
Mechanical Properties from Periodic Plane Wave QM Codes: The Challenge of the Flexible Nanoporous MIL-47(V) Framework
}

\author{
Danny E. P. Vanpoucke, ${ }^{1,2,3}$ Kurt Lejaeghere, ${ }^{1,3}$ Veronique Van Speybroeck, ${ }^{1}$ Michel Waroquier, ${ }^{1}$ and An Ghysels ${ }^{1,4}$ \\ ${ }^{1}$ Center for Molecular Modeling, Ghent University, Technologiepark 903, 9052 Zwijnaarde, Belgium \\ ${ }^{2}$ Department of Inorganic and Physical Chemistry, \\ Center for Ordered Materials, Organometallics and Catalysis (COMOC), \\ Ghent University, Krijgslaan 281 (S3), 9000 Gent, Belgium \\ ${ }^{3}$ These two authors contributed equally. \\ ${ }^{4}$ Corresponding author: an.ghysels@ugent.be, Phone: +32 92646563
}

(Dated: September 17, 2015)

\begin{abstract}
Modeling the flexibility of metal-organic frameworks (MOFs) requires the computation of mechanical properties from first principles, e.g. for screening of materials in a database, for gaining insight in structural transformations, and for force field development. However, this paper shows that computations with periodic density functional theory are challenged by the flexibility of these materials: guidelines from experience with standard solid state calculations cannot be simply transfered to flexible porous frameworks. Our test case, the MIL-47(V) material, has a large-pore and a narrow-pore shape. The effect of Pulay stress (cf. Pulay forces) leads to drastic errors for a simple structure optimization of the flexible MIL-47(V) material. Pulay stress is an artificial stress that tends to lower the volume and is caused by the finite size of the plane wave basis set. We have investigated the importance of this Pulay stress, of symmetry breaking, and of k-point sampling on (a) the structure optimization, and (b) mechanical properties such as elastic constants and bulk modulus, of both the large-pore and narrow-pore structure of MIL-47(V). We found that, in the structure optimization, Pulay effects should be avoided by using a fitting procedure, where an equation of state $\mathrm{E}(\mathrm{V})$ (EOS) is fit to a series of energy versus volume points. Manual symmetry breaking could successfully lower MIL-47(V)'s energy by distorting the vanadium-oxide distances in the vanadyl chains and by rotating the benzene linkers. For the mechanical properties, the curvature of the EOS curve was compared with the Reuss bulk modulus, derived from the elastic tensor in the harmonic approximation. Errors induced by anharmonicity, the eggbox effect, and Pulay effects propagate into the Reuss modulus. The strong coupling of the unit cell axes when the unit cell deforms expresses itself in numerical instability of the Reuss modulus. For a flexible material, it is therefore advisible to resort to the EOS fit procedure.
\end{abstract}

\section{INTRODUCTION}

Metal-organic frameworks (MOFs) present a recent class of materials which have been receiving growing interest over the last decade. ${ }^{1,2}$ These materials show properties akin to both solids and molecular systems since they consist of inorganic metal clusters, indicated as nodes, connected through organic molecules, indicated as linkers. The linker-and-node topology often results in porous, highly tunable frameworks. Having internal surface areas of $>1000 \mathrm{~m}^{2} \mathrm{~g}^{-1}$ and a chemical tunability through the choice of nodes and linkers makes MOFs versatile materials. As such, they attract much interest for application within catalysis, gas separation and gas storage. ${ }^{3-10}$

This interest is clearly mirrored in the large body of experimental work on these materials, and the steadily growing body of theoretical work. Due to the size and complexity of MOFs, theoretical work mostly uses forcefield based methods. ${ }^{5,7,9,11-15}$ It is very useful to be able to validate force-field findings with $a b$ initio methods as these do not depend on the specific choices made in the force field parametrization. Over the last few years also the amount of $a b$ initio calculations on MOFs has been growing, providing detailed insights into the fundamental physics and chemistry of these materials. ${ }^{9,16-23}$
An fascinating subclass of MOFs are the so-called breathing MOFs. ${ }^{23-27}$ These MOFs show reversible structural phase transitions that are accompanied by large variations in unit cell volume (up to $50 \%$ and more) under the influence of thermal, mechanical or chemical stimuli. This makes them great candidates for sensing applications. However, to build such applications, the breathing behavior needs to be thoroughly understood. Because barriers between the structural phases are rather small, high-accuracy methods, such as density functional theory (DFT), are required to properly describe the energyvolume relation governing the structural phase transition.

DFT has been well established in the solid-state community for many years, and has been found to accurately handle metallic, semiconducting and insulating materials, containing all elements of the periodic table. ${ }^{28}$ Experience with these dense solids has, over the years, led to some general guiding rules or intuitions regarding what is required for a solid-state calculation to have a certain level of accuracy. However, in contrast to these dense compounds, MOFs represent a significantly different class of materials. The large pores lead to very open structures and very low densities. In addition, the combination of metal(-oxide) nodes and organic linkers may lead to the emergence of low dimensional 
physics. ${ }^{16,20,21,23,29}$ Furthermore, both covalent and noncovalent interactions are essential to reproduce the equilibrium structure and thus also the unit cell. These interactions and the interplay between them are of fundamental importance to explain the breathing phenomena of some MOFs. ${ }^{30-33}$ In case of breathing MOFs, the structural flexibility gives rise to very flat potential energy surfaces, making structure optimization highly nontrivial.

These phenomena make it hard to find a consistent yet accurate approach to perform DFT calculations for breathing MOFs. MOFs are extended periodic frameworks, and hence DFT implementations in periodic codes is mandatory to construct the potential energy surface accurately. A variety of studies have appeared on DFT calculations of MOFs using various computational choices. It is however not fully clear how these choices influence the overall accuracy. For instance, several authors employ a fairly sparse grid in reciprocal space of $1 \times 1 \times 1 k$-point sampling to keep MOF computations feasible. ${ }^{18,34,35}$ Geometry optimizations are another critical point. Liu et al. optimized MIL-53 with a small $k$ point spacing $\left(0.05 \AA^{-1}\right)$ while keeping the unit cell parameters fixed, giving a geometry with remaining imaginary frequencies. ${ }^{36}$ Walker et al., on the other hand, optimized MIL-53 using a $4 \times 4 \times 4 k$-grid and maintained the crystal symmetry during the optimization process. ${ }^{30}$ Ortiz et al. published mechanical properties of several MOFs (among which MIL-47 and MIL-53) using $3 \times 3 \times 3$ $k$-grids. ${ }^{37}$ Some authors already indicated problems of numerical sensitivity for porous materials. Sauer et al., for instance, investigated the convergence and anharmonicity of frequency computations. ${ }^{38}$

In this work, we revisit guiding rules of standard solid-state computations, and investigate the influence of methodological aspects with regard to the accuracy of (a) structural and (b) mechanical properties of breathing MOFs such as elastic constants and bulk moduli. Aspects of interest are real- and reciprocal-space integration grids, Pulay stresses and symmetry.

As a case study, the MIL-47(V) MOF, will be used, as shown in Fig. 1a-c. ${ }^{39}$ This MOF belongs to the subclass of breathing MOFs, where it has a somewhat special status. Unlike other breathing MOFs with the same topology (e.g. MIL-53(Al), MIL-53(Cr),...), MIL-47(V) does not show breathing under thermal stimuli or the adsorption of gases. ${ }^{25,40,41}$ In contrast, MIL-47(V) shows breathing under significant mechanical pressure. ${ }^{23,26} \mathrm{Be}-$ cause of this more rigid nature than other breathing MOFs, MIL-47(V) is assumed to be better-behaved in the computations, and thus more suitable for investigating methodological aspects.

Section II gives a brief introduction on standard numerical methods used in ab initio solid-state physics, while Section III provides computational details of this work. In Section IV, we study the influence of the methods and aspects discussed in section II on the accuracy of the structural properties in our case study of the MIL-
47(V) MOF in a large-pore configuration. Guiding rules are derived for accurate calculation of the properties of breathing MOFs. These new guiding rules are then applied in Section V to generate a narrow-pore MIL-47(V) and allow the comparison of the physical properties of the large-pore and the narrow-pore configuration. Finally, in Section VI, the conclusions are presented.

\section{METHODOLOGY}

\section{A. Solving the Schrödinger equation for solids}

Bloch functions and $k$-points. According to Bloch's theorem, the eigenfunctions of the Schrödinger equation with a periodic potential are Bloch functions, labeled by the vector $\mathbf{k}$ in reciprocal space (a $k$-point) and an extra index $n$ for residual degeneracy (band index),

$$
\psi_{\mathbf{k} n}(\mathbf{r})=u_{\mathbf{k} n}(\mathbf{r}) e^{i \mathbf{k} \cdot \mathbf{r}},
$$

where $u_{\mathbf{k} n}(\mathbf{r})$ has the same periodicity as the periodic potential. In crystalline solids, the one-electron orbitals in the Hartree-Fock or Kohn-Sham formalism need to fulfill the same type of differential equations as the Schrödinger equation and can hence be written as Bloch functions as well. Only the Bloch functions with $\mathbf{k}$ originating from a single Brillouin zone are independent, so the $\mathbf{k}$-vectors may be limited to the first Brillouin zone.

To compute the physical properties of solids, integrals over the first Brillouin zone are often required. Such integrals are in practice approximated by a sum over discrete $k$-points, the so-called $k$-point sampling. The distribution scheme by Monkhorst and Pack, for example, creates an uniformly spaced $k$-point grid symmetric around the $\Gamma$ point and is often used. ${ }^{42} \Gamma$ point sampling considers a single Bloch function in the integral with reciprocal vector $\mathbf{k}=\mathbf{0}(1 \times 1 \times 1$ sampling of the Brillouin zone), while $N \times N \times N$ sampling considers $N^{3}$ Bloch functions with $\mathbf{k}$-vectors distributed over the first Brillouin zone. Symmetry allows one to consider only the symmetry-inequivalent $k$-points and to write the integrals over the first Brillouin zone as a weighted sum of these irreducible $k$-points. The number of irreducible $k$-points $N_{\text {irr }}$ hence determines the computational cost of a single self-consistent field ( $\mathrm{SCF}$ ) cycle.

Plane wave basis set and Pulay stress. The periodicity of the material makes the plane-wave (PW) basis set $e^{i \mathbf{G} \cdot \mathbf{r}}$ a suitable choice to expand the Bloch functions. Here the reciprocal wavevectors $\mathbf{G}$ are linear combinations of the reciprocal unit cell lattice vectors $\left(\mathbf{G}_{1}, \mathbf{G}_{2}, \mathbf{G}_{3}\right)$. The expansion of the periodic $u_{\mathbf{k} n}(\mathbf{r})$ yields

$$
\psi_{\mathbf{k} n}(\mathbf{r})=\sum_{\mathbf{G}} u_{\mathbf{k} n, \mathbf{G}} e^{i(\mathbf{k}+\mathbf{G}) \cdot \mathbf{r}}
$$

where the coefficients $u_{\mathbf{k} n, \mathbf{G}}$ are the Fourier transform of $u_{\mathbf{k} n}(\mathbf{r})$. 
(a) $\mathbf{L P}$

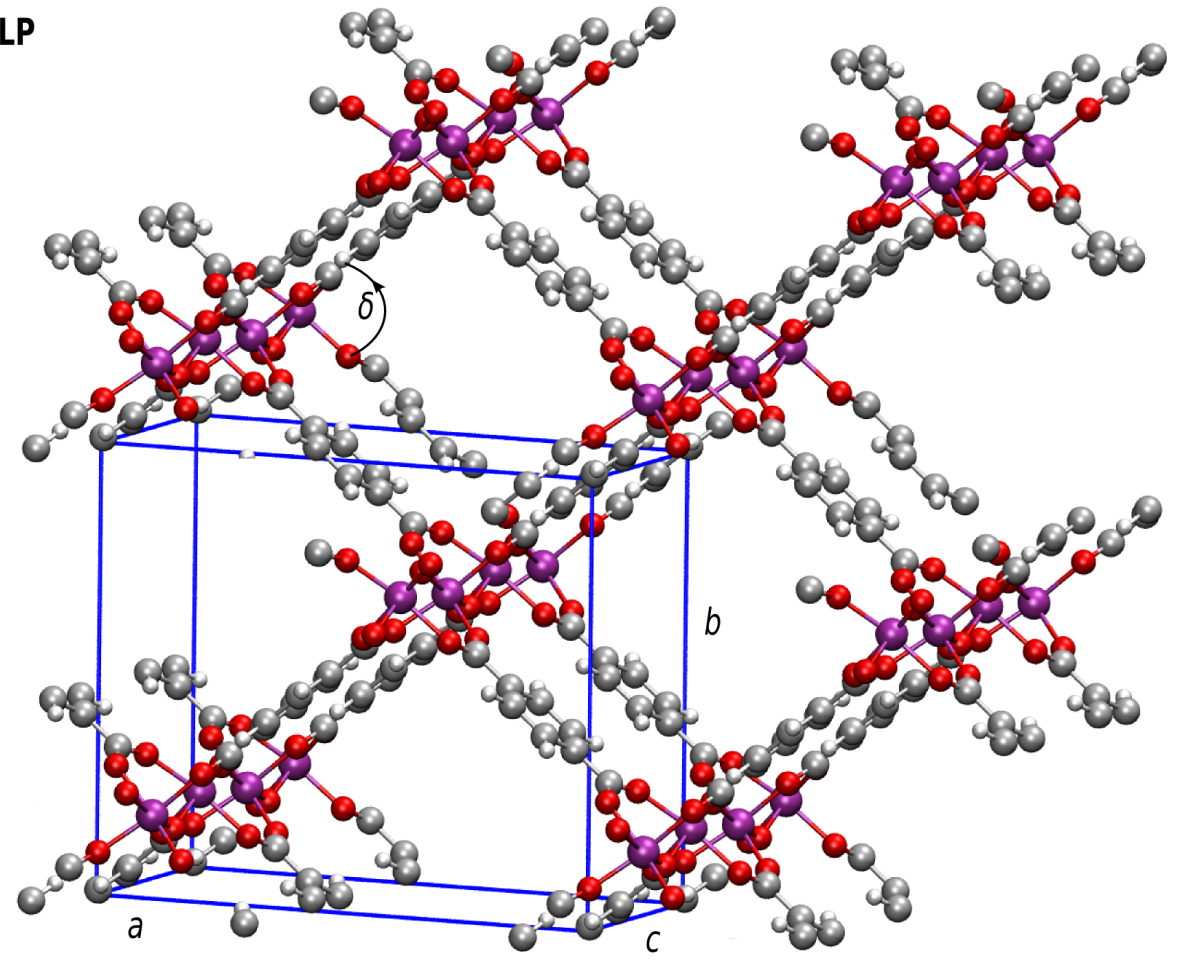

(b) NP

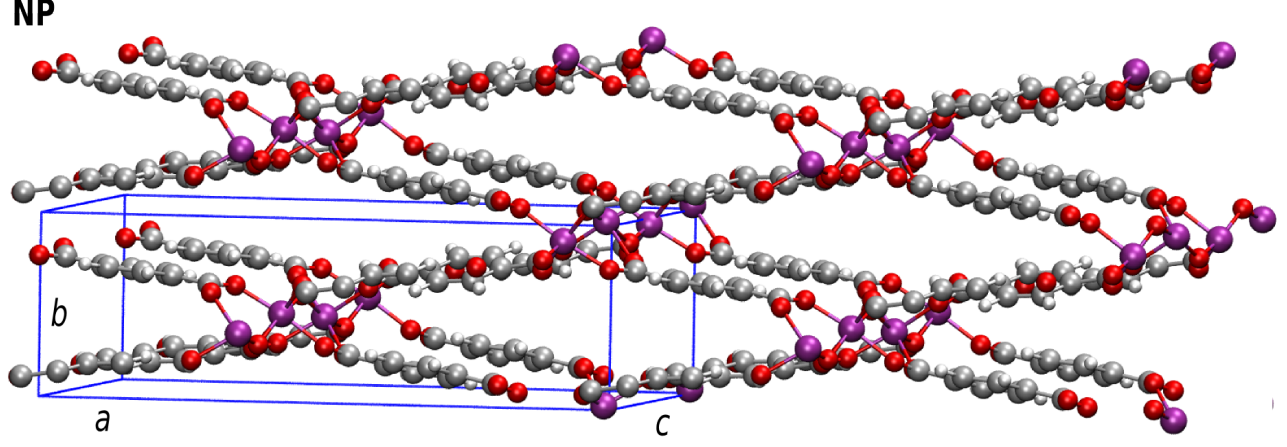

(c)

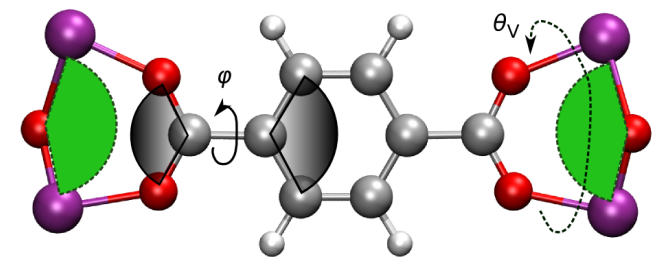

FIG. 1. (a,b) Unit cell of the large-pore (LP) and narrow-pore (NP) structure of MIL-47(V), with definition of opening angle $\delta$. (c) Definition of angles $\theta_{\mathrm{V}}$ and $\phi . \theta_{\mathrm{V}}$ is the dihedral angle between the VCV planes of opposite vanadyl chains and is a measure for their torsion. $\phi$ is the dihedral angle between the OCO plane and the terephthalate linker and is a measure for the planarity of the linker. Color code of atoms: grey $(\mathrm{C})$, red $(\mathrm{O})$, white $(\mathrm{H})$, pink $(\mathrm{V})$.

In practice, numerical applications are limited to a finite $\mathrm{PW}$ basis set $e^{i \mathbf{G} \cdot \mathbf{r}}$. The summation over reciprocal lattice vectors in Eq. 2 is therefore limited to $\mathbf{G}$ vectors whose length lies below a certain cutoff $G_{\text {cut }}$, determined by the cutoff energy: $\hbar^{2}|\mathbf{k}+\mathbf{G}|^{2} /\left(2 m_{e}\right) \leq$ $\hbar^{2} G_{\text {cut }}^{2} /\left(2 m_{e}\right)=E_{\text {cut }}$, for all G. In this way, $G_{\text {cut }}$ defines the resolution in real space as $\lambda_{\min }=2 \pi / G_{\text {cut }}$; fluctuations of $\psi_{\mathbf{k} n}(\mathbf{r})$ with shorter wavelengths are not taken into account in the basis set.

As this practical $\mathrm{PW}$ basis set is not complete, numerical inaccuracies appear. Even when the PW is that large that no effect can be noticed anymore in a static 


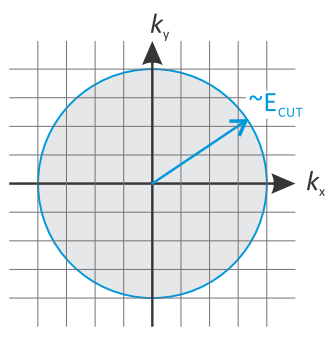

$E_{\text {cUt }}$ determines sphere in reciprocal space
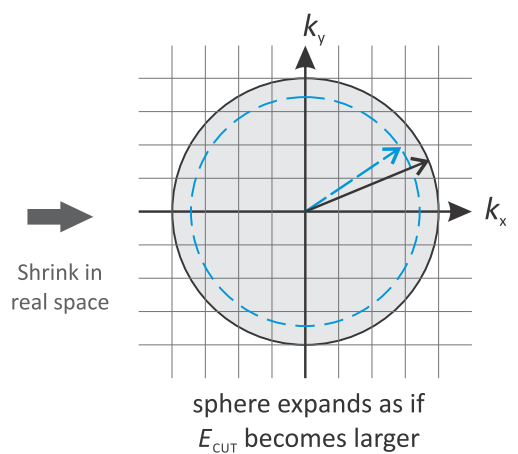

FIG. 2. The cutoff energy $E_{\text {cut }}$ determines the number of plane waves $N_{\mathrm{PW}}$ in the basis set, which cover a sphere in reciprocal space. Shrinking the unit cell in real space makes the sphere expand. This resembles an increase of the cutoff energy when $N_{\mathrm{PW}}$ is fixed, i.e. the Pulay effect.

energy calculation, the finite size of the PW basis set still causes an artificial Pulay stress, which affects optimizations of the crystal volume. As suggested by the name, Pulay stresses are similar to Pulay forces. The latter are caused by a finite atom-centered basis set, where basis functions are attached to the position of the nucleus, and are dragged along when the nucleus is displaced. If the basis set has a finite size, this creates an additional force on the nucleus, the Pulay force. Likewise, the PW basis set $e^{i \mathbf{G} \cdot \mathbf{r}}$ is linked to the unit cell dimensions through G. When the unit cell shrinks (expands), the size of the first Brillouin zone and the corresponding reciprocal lattice vectors $\mathbf{G}$ increase (decrease). The effect is a stress towards smaller volumes.

This can be understood from Fig. 2. Consider a reference volume and a PW basis set with $N_{\mathrm{PW}}$ plane waves, which covers a sphere in reciprocal space with a radius set by the cutoff energy $E_{\text {cut }}$. A volume contraction in real space corresponds to an expansion of the sphere in reciprocal space. This expansion resembles the effect of an increase in cutoff energy, i.e. as if the basis set were expanded (assuming for a moment complete $k$-point sampling). Because of the variational principle, this apparent basis set expansion will lower the energy. In other words, a volume contraction tends to lower the energy when the basis set is fixed. Taking a fixed finite number of plane waves $N_{\mathrm{PW}}$ thus creates an artificial tendency to reduce the volume in real space. This artifact is known as $\mathrm{Pu}-$ lay stress ${ }^{43-46}$ and causes volume optimizations, which typically operate with constant basis sets, to lead to too small volumes.

Integration on a real-space grid and eggbox effect. To combine both the reciprocal- and real-space parts into one set of Kohn-Sham equations, transformations between real and reciprocal space are required. In practice, this is done with a Fast Fourier Transform (FFT), which is an efficient implementation of the discrete Fourier transform, and which allows moving back and forth between discrete grids in real and reciprocal space. However, this may introduce numerical noise, since some high frequencies components, typically originating from the pseudopotentials and exchangecorrelation potential, cannot be transformed accurately between the real-space and reciprocal-space grid, as discussed in more detail in the Supp. Info. As a result, a small displacement of all atoms with respect to the grid gives a subtle change of the wavefunction, energy and forces. Only for a displacement over an entire grid spacing do these results remain invariant. Such periodic ripples as a function of the atomic shift are suitably named 'eggbox effect', after the periodic layout of cardboard boxes used to transport eggs.

\section{B. Structural properties of solids}

Equation of state. One of the key structural properties of a solid is its response to volume change. The energy profile as a function of volume is constructed in the following way. First, a series of starting structures is generated by uniformly straining the unit cell vectors to impose volume increments and decrements of up to a few percent of the reference volume. Each of these starting structures should be optimized while keeping the volume fixed; the ion positions and the unit cell shape are optimized. The resulting energies are gathered as the $E(V)$ profile (energy $E$ is expressed per unit cell).

Next, an equation of state (EOS) model $E_{\mathrm{EOS}}(V)$ is fitted to the computed $E(V)$ curve using a least-squares criterion. The commonly used Birch-Murnaghan EOS is only applicable to moderate volume changes, because it is derived from a third-order expansion of the free energy with respect to Lagrangian strain. ${ }^{47}$ For materials like soft MOFs, whose flexibility implies significant anharmonicity, we use the Vinet EOS, which is known to represent compression features much better (see discussion and Fig. S2 in Supp. Inf.). ${ }^{48,49}$ It is based on the universal binding-energy relation for solids (UBER) ${ }^{50}$ and depends on four material parameters: the minimum energy $E_{\min }$, the bulk modulus $B_{0}$, the derivative of the bulk modulus with respect to pressure $B_{0}^{\prime}=\partial B_{0} / \partial P$, and the volume $V_{\text {min }}$; all evaluated at the minimum energy point, at zero external pressure and zero temperature. If the cube root of the volume ratio $V / V_{\min }$ is denoted as $\eta=\sqrt[3]{\frac{V}{V_{\min }}}$, the Vinet EOS reads

$$
\begin{aligned}
E_{\mathrm{EOS}}(V)=E_{\min }+ & \frac{2 B_{0} V_{\min }}{\left(B_{0}^{\prime}-1\right)^{2}}\left[2-\left(5+3 B_{0}^{\prime}(\eta-1)-3 \eta\right)\right. \\
& \left.\times \exp \left(-\frac{3}{2}\left(B_{0}^{\prime}-1\right)(\eta-1)\right)\right]
\end{aligned}
$$

Using such an EOS fit makes it possible not only to determine elastic properties like $B_{0}$ (see further in Eq. 11) but also to determine $V_{\min }$ avoiding Pulay effects.

Finally, once $V_{\min }$ is found from the fit, the corresponding unit cell shape and ion positions are determined by 
optimizing the ion positions and unit cell shape while keeping the volume fixed at $V_{\min }$. This final structure will be referred to as the EOS structure. Its energy is denoted as $\tilde{E}_{\min }$ and can slightly differ from the fit value $E_{\text {min }}$.

Gamma-point phonons and thermodynamical quantities. To verify whether a geometry is a local minimum on the energy surface, a normal mode analysis (NMA) can be performed based on the Hessian. The Hessian matrix has dimension $3 N_{\text {at }} \times 3 N_{\text {at }}\left(N_{\text {at }}\right.$ number of atoms) and contains the second derivatives of the energy with respect to ion displacements evaluated at a stationary reference point on the energy surface, ${ }^{51}$

$$
H_{i j}=\left(\frac{\partial^{2} E}{\partial x_{i} \partial x_{j}}\right)_{0},
$$

where $i, j=1, \ldots, 3 N_{\text {at }}$. NMA is equivalent with a frozen phonon calculation: the Hessian equals the dynamical matrix when the periodicity of the phonons is set to one unit cell (also called $\Gamma$-point phonons). When the Hessian is a positive semidefinite matrix and the energy gradient is zero, the structure is a local minimum on the energy surface. On the other hand, when the Hessian has $n$ imaginary eigenvalues (frequencies), the structure is an $n$-fold saddle point, and a distortion of the reference geometry along the corresponding eigenvectors (normal modes) may push the structure towards a local minimum.

The phonon frequencies also serve as input in the vibrational partition function, from which several thermodynamic quantities may be derived, e.g. the zero-point energy (ZPE) and the vibrational finite-temperature corrections to the internal energy, entropy, and free energy. ${ }^{52}$

Stiffness tensor. The stiffness tensor $C$ is a generalization of the force constant $k$ in Hooke's law $F=k \Delta x$ to three-dimensional solids. In the linear regime it describes the stress response to strain of the unit cell,

$$
\overline{\bar{\sigma}}=C \cdot \overline{\bar{\epsilon}},
$$

where $\overline{\bar{\sigma}}$ is the Cauchy stress tensor and $\overline{\bar{\epsilon}}$ the strain tensor. We follow the definitions of the tensors as described in Ref. ${ }^{53}$. In the harmonic approximation, the clampedion stiffness tensor

$$
C_{k l}^{\text {clamp }}=\frac{1}{V_{0}}\left(\frac{\partial^{2} E}{\partial \epsilon_{k} \partial \epsilon_{l}}\right)_{0}
$$

is computed. It has dimension $6 \times 6$ in Voigt notation and contains the derivatives of the energy with respect to the elements of the strain tensor $\overline{\bar{\epsilon}}$, evaluated at a reference point with volume $V_{0}$, while keeping the atomic fractional coordinates fixed (hence the name clamped-ion). It can, for example, be computed by numerical differentiation of the stress tensor $\overline{\bar{\sigma}}$ at some strained structures, while keeping the fractional coordinates of the ions unaltered. An alternative method is energy-based and involves fitting the energy of strained structures to polynomials of $\epsilon_{i}$, similar to how an EOS is determined, and taking the second derivative of the polynomials at the reference point.
$C^{\text {clamp }}$ overestimates the rigidity of the material, because, in reality, the ions will relax when the material is strained. The relaxed ion tensor $C^{\text {relax }}$ is therefore computed too, by relaxing the ion positions. This can be done manually, by optimizing the ion positions at each strained structure separately. It includes the anharmonicities of the ion response. Alternatively, the ion response correction can also be applied to $C^{\text {clamp }}$ in the harmonic approximation, neglecting anharmonicities, in the following procedure. First, the 'extended Hessian' with six additional rows and columns is computed. It contains the Hessian $H$ and $C^{\text {clamp }}$ as diagonal blocks and the forceresponse internal strain tensor $B$ as off-diagonal block, ${ }^{53}$

$$
B_{i, k}=\left(\frac{\partial^{2} E}{\partial x_{i} \partial \epsilon_{k}}\right)_{0},
$$

where the mixed partial derivatives in this $3 N_{\mathrm{at}} \times 6 \mathrm{ma}-$ trix are evaluated at the reference structure. $B$ is, for example, computed by numerical differentiation of either the forces in a strained cell (with constant fractional coordinates), or the stresses due to displaced ions (in a constant cell). Averaging over these two approaches can improve numerical accuracy. Next, this ionic response is added as a correction term ${ }^{53}$ to $C^{\text {clamp }}$, yielding $C^{\text {relax }}$, denoted in short as $C$,

$$
C=C^{\text {relax }}=C^{\text {clamp }}-\frac{1}{V_{0}} B^{T} \cdot H^{-} \cdot B
$$

where $H^{-}$is the pseudo-inverse of the Hessian. ${ }^{53}$ This expression resembles the general principle of Vibrational Subsystem Analysis (VSA), where the subsystem degrees of freedom relax adiabatically along the normal modes when the environment degrees of freedom are manipulated. ${ }^{54-56}$ The correction term in Eq. 8 may be regarded as an application of VSA: the unit cell parameters are subsystem coordinates, and the ionic positions are environment coordinates. A note of caution is in order, however: the accuracy of $C^{\text {relax }}$ is particularly sensitive to the low eigenvalues of the Hessian because of the appearance of its pseudo-inverse in Eq. 8.

The elements of the stiffness matrix represent the resistance of the material to different deformations in different directions. On the other hand, it can also be useful to consider the eigenvalues of $C$. The lowest and the highest eigenvalue indicate the easiest (so-called 'soft mode') and hardest deformation mode, respectively. ${ }^{37,57,58}$

Bulk modulus. The bulk modulus describes the overall resistance to strain or stress. Several definitions exist, because volume change may be realized in several ways. Close to the reference volume, bulk moduli can be computed from the stiffness tensor, giving 'harmonic' estimates. Alternatively, the bulk modulus directly relates to the second derivative of the equation of state curve $E_{\mathrm{EOS}}(V)$, giving the EOS estimate. As non-cubic unit cells are much less commonly discussed in literature than cubic systems, we briefly revisit different measures of the bulk modulus and their interpretation. 
(1) The Voigt modulus $K_{V}$ is an average of the upper left corner of the stiffness tensor,

$$
\begin{aligned}
K_{V} & =\frac{1}{9} \sum_{i, j=1}^{3} C_{i j} \\
& =\frac{1}{9}\left(C_{11}+C_{22}+C_{33}+2 C_{12}+2 C_{13}+2 C_{23}\right) .
\end{aligned}
$$

It describes the average response of the linearly elastic tensile stress to isotropic volumetric strain $(\overline{\bar{\epsilon}} \rightarrow \overline{\bar{\sigma}}=$ $C \cdot \overline{\bar{\epsilon}})$.

(2) The Reuss modulus $K_{R}$ relates to the inverse of the average of the upper left corner of the compliance matrix $S\left(S=C^{-1}\right)$,

$$
\begin{aligned}
K_{R} & =\left(\sum_{i, j=1}^{3} S_{i j}\right)^{-1} \\
& =\left(S_{11}+S_{22}+S_{33}+2 S_{12}+2 S_{13}+2 S_{23}\right)^{-1} .
\end{aligned}
$$

It corresponds to volume changes induced by isotropic pressure under the assumption of a linearly elastic material $(\overline{\bar{\sigma}} \rightarrow \overline{\bar{\epsilon}}=S \cdot \overline{\bar{\sigma}})$ and follows from imposing an isotropic stress tensor $\sigma_{i j}=\sigma \delta_{i j}$. The Reuss modulus may also be derived using the generalized VSA principle ${ }^{56}$ (see discussion Eq. 8) by projecting out deformation modes that keep the volume $V$ constant. This shows that the Reuss modulus may indeed be interpreted as the response to a volume change without specifying how it is realized, allowing changes in cell shape to reduce anisotropic stresses.

(3) In the Vinet EOS, Eq. 3, $B_{0}$ expresses the resistance of the material against volume change and can be calculated analytically as

$$
B_{0}=\left(V \frac{\partial^{2} E}{\partial V^{2}}\right)_{\min }=-V_{\min }\left(\frac{\partial P}{\partial V}\right)_{\min },
$$

evaluated at the minimum-energy volume $V_{\min }$. How the volume change is realized, e.g. by isotropic stretch or uniaxal stretch, is not specified, only that the minimumenergy geometry is taken at each volume. In the case of our $a b$ initio calculations of with periodic boundary conditions representing a single crystal, $B_{0}$ should therefore be compared with the Reuss modulus $K_{R}$.

\section{COMPUTATIONAL DETAILS}

Calculation of properties of MOFs with plane-wave DFT is prone to numerical inaccuracies and requires extreme care to obtain reliable results. In Secs. IV and V, we demonstrate this for MIL-47(V). The starting structures are taken from the CIF database of the Cambridge Crystallographic Data Centre. ${ }^{59}$ For the largepore phase, we use the structure with CCDC code 846906 (dubbed 'geom1'), derived from a combined experimental/theoretical study by Maurin and co-authors, ${ }^{26}$ and the open structure (CCDC code: 166785, 'geom2') measured from the empty material by the group of Férey. ${ }^{39}$ For the narrow-pore phase, we use the closed form of Maurin and co-authors (CCDC code: 846907, 'geom3'). ${ }^{26}$ Table I lists the cell parameters. Each unit cell contains 72 atoms in total. Energies will be expressed per unit cell.

All calculations are performed using the projector augmented wave (PAW) method as implemented in the Vienna Ab Initio Simulation Package (VASP). For the C and $\mathrm{O}$ atoms, the $2 s$ and $2 p$ electrons are considered as valence electrons, while for the $\mathrm{V}$ atoms, the $3 p$, $3 d$ and $4 s$ electrons are considered as valence electrons. The exchange and correlation behavior of the electrons is modeled with the Generalized Gradient Approximation (GGA) functional constructed by Perdew, Burke and Ernzerhof (PBE), ${ }^{60}$ which is known to provide reliable predictions for a wide range of solids and properties. ${ }^{61}$ Dispersion interactions between the organic linkers are well known to be of importance to correctly describe the MOF structure. ${ }^{30,62}$ Van der Waals interactions are modeled using the Grimme DFT-D3 corrections ${ }^{63}$ with Becke-Johnson damping ${ }^{64}$ and a cutoff radius of $50 \AA$. Spin polarization is taken into account, and the total magnetic moment per unit cell is set to 4 , as every vanadium atom has one unpaired electron.

Although subsequent sections discuss the sensitivity of the results to a number of numerical parameters, there are also some common settings. First, the kinetic energy cutoff $E_{\text {cut }}$ is set to $500 \mathrm{eV}$, corresponding to a largest wavevector $G_{\text {cut }}=11.0 \AA^{-1}$, and the criterion for energy convergence for a single SCF cycle is put at $10^{-8} \mathrm{eV}$. A Gaussian smearing scheme with a smearing factor of $\sigma=0.05 \mathrm{eV}$ is used. The number of grid points $N_{\mathrm{FFT}}$ in the FFT is set to twice the number of plane waves in each direction to avoid FFT wrapping errors in reciprocal space. Finally, when optimizing the geometry, an energy convergence criterion of $10^{-7} \mathrm{eV}$ is used, leading to the largest forces being only a $3.2 \mathrm{meV} / \AA$ or less after optimization.

For phonon calculations, ionic displacements of $0.01 \AA$ are applied, while for the determination of elastic constants, the strain tensor elements are set to $1 \%$. The energy profile, on the other hand, is generated by changing the equilibrium volume up to $\pm 4 \%$ in steps of $1 \%$, to which a Vinet EOS is fitted.

\section{STRUCTURE DETERMINATION OF MIL-47(V)}

Because of their particular bonding character and the small energy differences involved in their structural transformations, breathing MOFs are strongly sensitive to numerical effects that do not play a significant role in other types of materials. The particular morphology of breathing MOFs gives rise to relatively flat potential energy surfaces. Different structural configurations barely dif- 
TABLE I. Starting structures of MIL-47(V) for its large-pore shape (geom1 and geom2) and narrow-pore shape (geom 3): lattice constants (in $\AA$ ), lattice angles (in ${ }^{\circ}$ ), volume (in $\AA^{3}$ ), and symmetry.

\begin{tabular}{lrrr}
\hline & geom1 & geom2 & geom3 \\
\hline$a$ & 16.6568 & 16.1433 & 21.117 \\
$b$ & 13.5806 & 13.9392 & 6.710 \\
$c$ & 6.7393 & 6.8179 & 6.717 \\
$\alpha$ & $90^{\circ}$ & $90^{\circ}$ & $90^{\circ}$ \\
$\beta$ & $90^{\circ}$ & $90^{\circ}$ & $114.41^{\circ}$ \\
$\gamma$ & $90^{\circ}$ & $90^{\circ}$ & $90^{\circ}$ \\
$V$ & 1524.5 & 1534.2 & 866.7 \\
space group & Imma & Pnma & $\mathrm{C} 2 / \mathrm{c}$ \\
CCDC & 846906 & 166785 & 846907 \\
\hline
\end{tabular}

fer in energy and are separated by low energy barriers, which are prone to large inaccuracies. These structural conformations and barriers serve as input in thermodynamic models for describing the breathing behavior, ${ }^{14,65}$ so their accurate determination is essential. In contrast, in other materials with a more coarsely shaped energy surface, the errors have less impact on the processes occuring on the potential energy surface. We will demonstrate how numerical inaccuracies may have an effect on the overall behavior of the breathing MOF. MIL-47(V) is a good prototype for this type of investigation, as it shows all features inherent to a flat energy surface as sketched above. In the following subsections the sensitivity of properties of the large-pore MIL-47(V) phase will be discussed.

\section{A. Energy convergence test (single point)}

In Table II, static energy calculations are performed on the two geometries geom 1 and geom 2 of Table I as a function of the $k$-point set. Since our interest in the energy is mainly limited to geometry optimizations and crystal deformations, we particularly require energy differences to be well converged. When only considering $N \times N \times N$ $k$-grids, a $6 \times 6 \times 6 k$-point set allows convergence up to $0.1 \mathrm{meV}$ at a cost of 27 irreducible $k$-points, which may serve as the reference in Table II. According to this convergence test, the $\Gamma$-point set $(1 \times 1 \times 1)$ performs poorly both in absolute energy and in energy difference between geom1 and geom2, indicating that errors do not cancel out.

Since the $a$ and $b$ lattice vectors are longer than the $c$ vector, we also consider some choices $N_{a} \times N_{b} \times N_{c}$ with $N_{a}=N_{b}<N_{c}$ to ensure a constant $k$-point density in each direction: $\Delta k \approx 2 \pi / a / N_{a} \approx 2 \pi / b / N_{b} \approx 2 \pi / c / N_{c}$ ( $a \approx b>c$ for MIL-47). We moreover require a very stringent convergence of energy differences (up to a few times $0.1 \mathrm{meV}$ per unit cell at most), since the large and narrow pore structure are separated by a small energy barrier. With six or more $k$-points along the shortest axis $c=6.9 \AA$, the energy is converged within $0.1 \mathrm{meV}$.
Because the other lattice constants $a$ and $b$ are larger than $c$, a $k$-point set of $2 \times 2 \times 6$ provides an accurate approximation of the reference $6 \times 6 \times 6$ energy at a much lower computational cost, and we therefore use this $k$ grid in the remainder of this work for the geometry optimization, phonon calculation, and flexibility analysis. For the interested reader, we provide computed data in the Supplementary Information for the smaller $k$-point sets $1 \times 1 \times 1$ and $2 \times 2 \times 2$.

The eggbox effect gives energy fluctuations as a function of the real space grid position. As shown in the Supporting Information, the $2 \times 2 \times 6$ energy fluctuates by about $0.1 \mathrm{meV}$, which represents the limit of the the energy accuracy.

\section{B. Structure optimization}

In this discussion, structure geom 1 is selected as the starting structure of the geometry optimization, because it has a lower energy than geom2. A material has not only $3 N_{\text {at }}$ coordinates describing the ion positions but also six coordinates $(a, b, c, \alpha, \beta, \gamma)$ describing the unit cell shape and volume. These degrees of freedom can be relaxed stepwize, by first relaxing the ion positions only, then also the unit cell shape, and finally the unit cell volume as well. The effect of the relaxation on the energy is visualized in Fig. 3a and values are given in Table III. Starting from the initial structure geom1, the ion geometry optimization-where only the ion coordinates are allowed to relax-lowers the energy with several tens of $\mathrm{eV}$. In the shape optimization, wherein besides the ion positions the shape of the unit cell is relaxed as well while the cell volume is kept fixed, the energy is further reduced by a few tens of meV. In the fully relaxed optimization, the volume is relaxed as well, thus relaxing all $3 N_{\text {at }}+6$ degrees of freedom. We observe that in this case the MIL47(V) structure shrinks to its NP shape. This is caused by the Pulay stress, as the optimization employs a constant yet finite basis set, pushing the structure towards smaller volumes (see also further).

To avoid these Pulay effects and retrieve a local minimum for the LP phase, the EOS profile can be constructed manually. A series of structures at distinct volumes is created with a shape optimization (i.e., keeping the volume fixed), as described in Section IIB. Fitting the Vinet equation of state (value of four parameters available in the Supporting Information) and reoptimizing at fixed volume indeed predicts a local minimum in the LP shape at $V_{\min }=1554.1 \AA^{3}$ and $\tilde{E}_{\min }=-539.9326 \mathrm{eV}$, which lies several meV below the shape structure.

In the following, three effects are discussed that influence the geometry optimization: the abovementioned Pulay stress, $k$-point sampling, and symmetry breaking. We moreover briefly discuss EOS fitting errors.

(a) Pulay stress. It is remarkable that the fully relaxed optimization yields a NP structure with higher energy than the shape optimized LP structure. This 
TABLE II. Energy convergence with increasing $k$-point set for two geometries (geom1 and geom2) and their difference in energy (diff). The van der Waals corrected ground state energy $E$ is expressed with respect to the $6 \times 6 \times 6$ reference value: $\delta E^{\text {geom1 }}=E^{\text {geom1 }}-E_{666}^{\text {geom1 }}$ and $\delta E^{\text {geom2 }}=E^{\text {geom2 }}-E_{666}^{\text {geom2}}$. For the set labeled with a star, the SCF did not converge after 200 cycles. $N_{\text {irr }}$ is the number of irreducible $k$-points, with symmetry turned on (sym) or off (nosym) in VASP.

\begin{tabular}{|c|c|c|c|c|c|}
\hline \multirow[t]{2}{*}{$k$-points } & \multicolumn{2}{|c|}{$N_{\text {irr }}$} & \multicolumn{3}{|c|}{$\delta E(\mathrm{meV})$} \\
\hline & sym & nosym & geom1 & geom2 & diff \\
\hline $1 \times 1 \times 1$ & 1 & 1 & 2216.7 & 1406.1 & 810.6 \\
\hline $2 \times 2 \times 2$ & 1 & 4 & -28.1 & -27.5 & -0.5 \\
\hline $3 \times 3 \times 3$ & 8 & 14 & 17.8 & 2.0 & 15.8 \\
\hline $4 \times 4 \times 4$ & 8 & 32 & 0.7 & -0.2 & 0.9 \\
\hline $5 \times 5 \times 5$ & 27 & 63 & 0.0 & 0.0 & 0.1 \\
\hline $6 \times 6 \times 6$ & 27 & 108 & 0 & 0 & 0 \\
\hline $1 \times 1 \times 2$ & 1 & 1 & -24.4 & -19.2 & -5.2 \\
\hline $2 \times 2 \times 4$ & 2 & 8 & 0.9 & -0.1 & 1.1 \\
\hline $3 \times 3 \times 6$ & 12 & 27 & 0.0 & 0.1 & -0.1 \\
\hline $1 \times 1 \times 3$ & 2 & 2 & $44.5^{*}$ & 9.9 & 34.6 \\
\hline $2 \times 2 \times 3$ & 2 & 6 & 17.9 & 2.0 & 15.9 \\
\hline $2 \times 2 \times 6$ & 3 & 12 & 0.0 & -0.1 & 0.2 \\
\hline $6 \times 6 \times 6$ & & & $E_{666}^{\text {geom1 }}(\mathrm{eV})$ & $E_{666}^{\text {geom2 }}(\mathrm{eV})$ & $\operatorname{diff}(\mathrm{eV})$ \\
\hline & & & -533.2859 & -529.6710 & 3.6148 \\
\hline
\end{tabular}

TABLE III. Geometry optimizations of the LP unit cell, starting from geom1. The energy (in meV) is expressed with respect to the $E O S$ energy with $2 \times 2 \times 6 k$-points: $\delta E=E-\tilde{E}_{\min }^{226}$ with $\tilde{E}_{\min }^{226}=-539.9326 \mathrm{eV}$. It was verified whether the geometry is a local minimum ( $\mathrm{min}$ ) or a saddle point (sp) by computing and diagonalizing the Hessian. The volume is given per unit cell (in $\AA^{3}$ ), the unit cell lengths $a, b, c$ (in $\AA$ ), the vanadyl chain angle $\theta_{V}$ (in ${ }^{\circ}$ ), and the benzene rotation angle $\phi$ (in ${ }^{\circ}$ ); see definition in Fig. 1. Unit cell dimensions as used by Ortiz et al. in the CRYSTAL package are given for comparison. ${ }^{37}$ The upper table displays the effects of successive optimization steps, while the lower table highlights three effects on the optimization: Pulay stress (fully relaxed), imposing symmetry, and $k$-point set.

\begin{tabular}{|c|c|c|c|c|c|c|c|c|c|c|}
\hline \multicolumn{11}{|c|}{ Successive optimizations } \\
\hline & $k$-points & $\delta E$ & & $V$ & $a$ & $b$ & $c$ & $\theta_{V}$ & $\phi$ & \\
\hline geom1 & $2 \times 2 \times 6$ & 6646.3 & & 1524.5 & 16.657 & 13.581 & 6.739 & 0 & 0 & \\
\hline ion & $2 \times 2 \times 6$ & 46.5 & $\mathrm{sp}$ & 1524.5 & 16.657 & 13.581 & 6.739 & 0 & 0 & \\
\hline shape & $2 \times 2 \times 6$ & 9.7 & $\min$ & 1524.5 & 16.865 & 13.241 & 6.827 & 8 & $1-8$ & \\
\hline EOS & $2 \times 2 \times 6$ & 0 & $\min$ & 1554.1 & 16.394 & 13.854 & 6.842 & 8 & $1-7$ & \\
\hline Ortiz $^{37}$ & $3 \times 3 \times 3$ & & & 1524.1 & 16.05 & 13.98 & 6.79 & & & \\
\hline \multicolumn{11}{|c|}{ Effects on optimizations } \\
\hline effect & & $k$-points & $\delta E$ & & $V$ & $a$ & $b$ & $c$ & $\theta_{V}$ & $\phi$ \\
\hline tress ful & y relaxed & $2 \times 2 \times 6$ & 82 & $\mathrm{sp}$ & 859.9 & 19.662 & 6.435 & 6.797 & 12 & 12 \\
\hline EC & S sym & $2 \times 2 \times 6$ & 338 & $\mathrm{sp}$ & 1506.9 & 17.062 & 13.162 & 6.710 & 0 & 0 \\
\hline EC & & $1 \times 1 \times 1$ & 870 & $\min$ & 1594.9 & 15.504 & 14.588 & 7.052 & 10 & $18-22$ \\
\hline EC & & $2 \times 2 \times 2$ & -84 & $\min$ & 1547.5 & 16.342 & 13.889 & 6.818 & 0 & 2 \\
\hline EC & & $2 \times 2 \times 6$ & 0 & $\min$ & 1554.1 & 16.394 & 13.854 & 6.842 & 8 & $1-7$ \\
\hline
\end{tabular}

means that the conjugate gradient optimizer was able to climb a slope and overcome the barrier in the $E(V)$ profile between the LP and NP structures. The PW basis set depends on the unit cell dimensions. Following the Hellmann-Feynman theorem, the energy change due to straining the unit cell has then two components, one originating from the derivative of the Hamiltonian, and another one originating from the change in basis set when the plane waves in the basis set are strained. The latter is the Pulay stress. All computed stresses are distorted by the Pulay stress, which is an artificial stress towards smaller volumes (see Section II A). While we would normally expect that a fully relaxed optimization invokes a very small volume change with respect to the shape optimization, we observe here that MIL- $47(\mathrm{~V})$ undergoes a drastic transformation from the LP to the NP shape with a significant volume drop.

This drastic effect is visualized in Fig. 4. According to the EOS optimization, the LP is a minimum energy structure of the $E(V)$ profile, which corresponds to a zero pressure at the LP volume. In Section V, the NP is shown to be a minimum energy structure of the $E(V)$ profile as well. The energy profile must therefore have two minima separated by a barrier, at which the pressure $P$ is zero (see Fig. 4). The fully relaxed optimization is driven by pressure, which is unfortunately affected by the Pulay stress. This Pulay stress, which varies only slightly as a function of volume ${ }^{46}$, shifts the pressure curve $P(V)$ downwards, such that the apparent pressure is no longer zero at the LP structure. Pulay stress thus seems to elim- 
a)

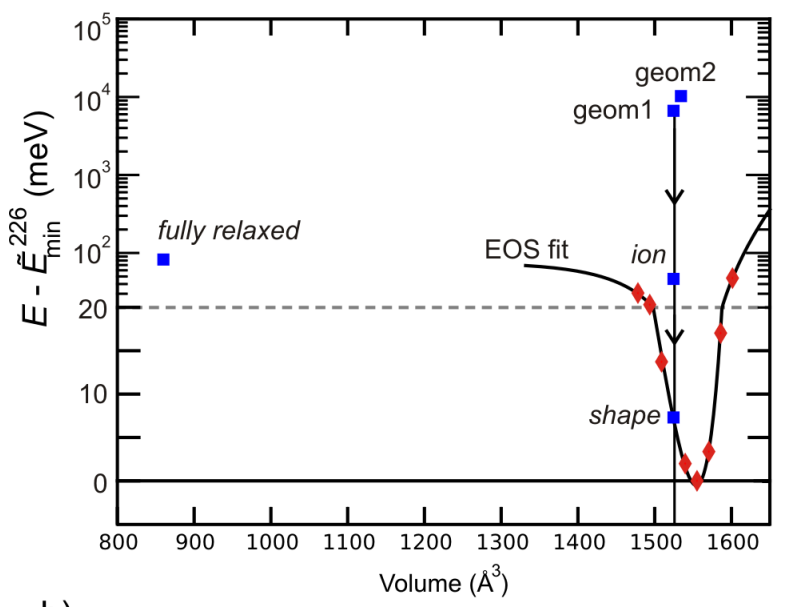

b)
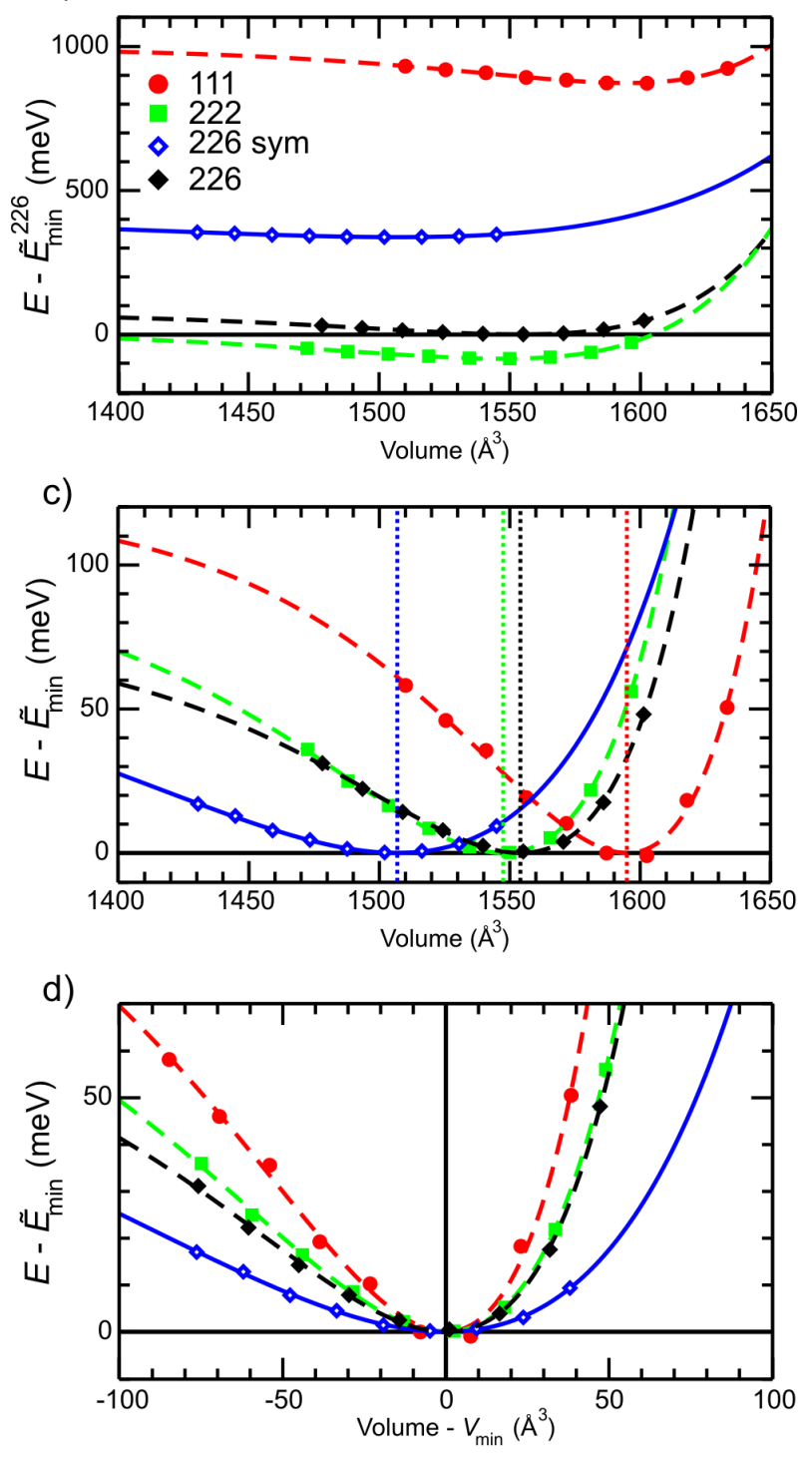

FIG. 3. Structure optimization of MIL-47(V). (a) Energy and volume of the starting geometries geom1 and geom2, ion optimization, shape optimization, fully relaxed optimization (blue squares). The Vinet EOS is fit (black line) to a set of $E\left(V_{i}\right)$ points (red diamonds), see Section IIB. Energy scale is linear (bottom part) and logarithmic (top part). (b-d) The EOS is fit (lines) to a set of strained structures (symbols). Effect of Pulay stress, symmetry, and $k$-point sampling: on the energy $E_{\min }(\mathrm{b})$, on the volume prediction $V_{\min }(\mathrm{c})$, and on the curvature $B_{0}(\mathrm{~d})$.

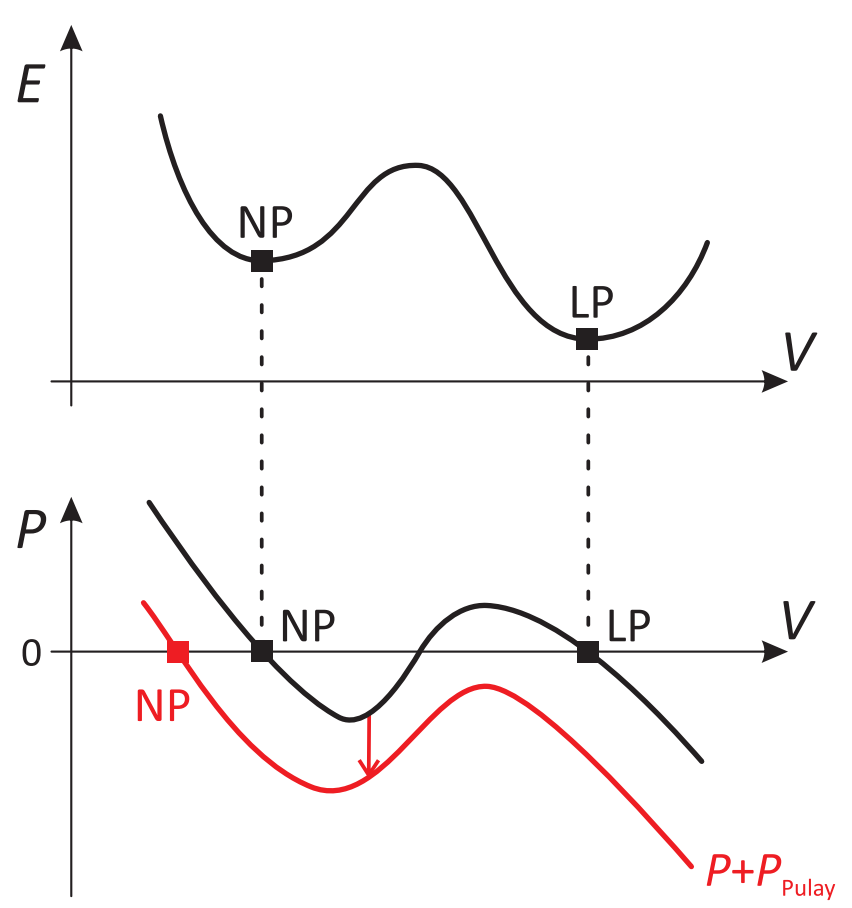

FIG. 4. In a fully relaxed optimization, Pulay stress pushes the structure out of the LP phase towards the NP phase. The energy profile $E(V)$ has two minima and a barrier, corresponding to zeros of the pressure profile $P(V)$. Pulay stress, approximately a constant, shifts the pressure profile downwards, such that the LP volume is no longer detected as a local minimum in the pressure-driven fully relaxed optimization.

inate the existence of the shallow LP minimum, pushing the system towards the NP structure.

The Pulay stress tensor is accessible by performing a stress calculation at the predicted EOS minimum-energy structure $V_{\min }$, where the stress tensor should be zero in the infinite basis set limit. The deviation from zero is the Pulay stress tensor, and in the case of LP MIL$47(\mathrm{~V})$, it is found to be isotropic, lying between -0.10 and $-0.14 \mathrm{GPa}$, depending on the symmetry and the used $k$-point set. For the $E O S$ structure with a $2 \times 2 \times 6 k$ grid, the Pulay stress amounts to $-0.125 \mathrm{GPa}$. This value is of the same order of magnitude as calculated transition pressures between the large- and narrow-pore phase from literature (82-125 MPa), ${ }^{23}$ which indeed supports the proposed mechanism shown in Fig. 4.

An additional note must be made about the accuracy of the found narrow-pore structure. At first sight, the fully relaxed structure with a volume of $859.9 \AA^{3}$ suggests that this NP shape is a second meta-stable state of MIL$47(\mathrm{~V})$ that has not been observed experimentally under ambient conditions. However, the comparison is of limited value because of the temperature difference between calculations (at $T=0 \mathrm{~K}$ ) and experiments (at finite temperature). Moreover, the fully relaxed optimization has 
been performed with the $2 \times 2 \times 6 k$-point set, which was only proven to be adequate for the description of the LP shape (Table II). As the unit cell dimensions of the NP shape are approximately $19.7 \times 6.4 \times 6.8 \AA^{3}$, the number of two $k$-points along the $b$-axis is probably too low, and needs to be enlarged. Our results based on the $2 \times 2 \times 6$ $k$-point set are thus not accurate enough to associate the NP shape to a local minimum or the global minimum at $0 \mathrm{~K}$. This item will be further addressed in Section V.

(b) $k$-point set. To highlight the sensitivity of the structure optimization to $k$-point sampling, the EOS profiles are constructed for the $1 \times 1 \times 1$ and $2 \times 2 \times 2 k$-point sets as well. Fig. 3b confirms that the energy is not converged with these limited $k$-point sets, as the curves do not coincide with the reference $2 \times 2 \times 6 k$-point set. This poor performance was to be expected from our $k$-point convergence test in the previous subsection (Table II). Moreover, the predicted volume deviates, as visualized in Fig. 3c, and the curvature (cf. bulk modulus) of the EOS profile is not well reproduced with limited $k$-point sets either, as shown in Fig. 3d. Apparently, one cannot rely on cancelation of errors when using too limited $k$ point sets in structure optimizations on such a shallow energy surface. The $k$-point convergence test for the energy gives a guideline for the minimal $k$-point set, here $2 \times 2 \times 6$, and this set should be used when doing a structure optimization as well.

(c) Symmetry breaking. Exploiting symmetry reduces the number of irreducible $k$-points from 12 to 3 when using a $2 \times 2 \times 6 k$-grid for MIL-47(V) (Table II) and considerably lowers the computational cost of the optimization (except for the subsequent phonon calculation using finite differences, as symmetry is then broken during ion displacements). However, imposing symmetry may also lead to an incomplete scanning of the potential energy surface, overlooking lower-symmetry structures. The effect of symmetry is therefore tested by imposing the initial geom1 symmetry (Imma) in the $2 \times 2 \times 6$-sym optimization.

Imposing a symmetry constraint shifts the resulting $2 \times 2 \times 6$-sym EOS profile $338 \mathrm{meV}$ above the reference profile (see Fig. 3b, Table III). The reason is that the symmetry constraint prevents linker rotation and vanadium chain twists to occur during the optimization: the predicted $2 \times 2 \times 6$-sym EOS has all vanadiums aligned and highest symmetry for the linkers. The structure is not a true minimum but a saddle point with some imaginary frequencies (Section IIB). Visualization of the corresponding eigenmodes indicates that these saddle point frequencies would induce a zig-zag in the vanadium chain and slightly tilted linkers, thus breaking the Imma symmetry.

Moreover, the $2 \times 2 \times 6$-sym profile predicts a deviating volume in Fig. 3c and deviating curvature in Fig. 3d. This is an indication that linker mobility and vanadyl chain distortions should be taken into account when the prediction of accurate energy curves is the objective.

Note that allowing symmetry breaking in the optimiza- tion run not necessarily implies that the implemented geometry optimizer will break symmetry; even without imposing the initial symmetry, the structures remain highly symmetric in most cases. In our shape optimization, for instance, a first run gave a saddle point with imaginary frequencies representing linker rotations, despite not having constrained the symmetry. Ions were distorted manually along the lowest eigenmodes to create the starting structure of the second run, which ultimately led to a structure without any imaginary frequencies. Getting stuck in a saddle point is hard to avoid in gradient based geometry optimizers. The force along the imaginary frequency displacement vector is zero in a saddle point, and an the implementation based on symmetry will not even generate any numerical noise on this force that could make the linkers rotate, such that the starting symmetry is maintained during the whole geometry optimization.

The change in symmetry through linker rotations is observable when measuring the dihedral angles $\theta_{\mathrm{V}}$ and $\phi$ (definition in Fig. 1). These dihedral angles are planar in the initial structure geom1. The angles can remain zero in the optimization to a saddle point, but to find a true minimum state, these dihedral angles need to deviate slightly from zero. Moreover, not all dihedral angles in a given structure are exactly equivalent: despite the topological equivalence of linkers and V-chains, their optimized dihedral angles are not all equal, meaning that even more symmetry has been broken. On the other hand, the unit cells in Table III are all orthorhombic (with deviations of less than $1^{\circ}$ ), so the symmetry of the Bravais lattice remains. Symmetry breaking through vanadyl chain distortions in the narrow-pore shape of MIL-47(V) will be discussed in Section V.

(d) EOS fitting errors. We finally assess the influence of the quality of the Vinet fit on the EOS parameter estimations. The fit to Eq. 3 is performed by minimizing the root mean square deviation (rmsd) between the computed VASP energies $E\left(V_{i}\right)$ and the EOS model energies $E_{\mathrm{EOS}}\left(V_{i}\right)$ at a series of volumes $V_{i}$,

$$
\operatorname{rmsd}=\sqrt{\frac{\sum_{i=1}^{i=9}\left(E\left(V_{i}\right)-E_{\mathrm{EOS}}\left(V_{i}\right)\right)^{2}}{9}} .
$$

Taking into account 9 volume points $V_{i}$, the rmsd is found to vary between 0.2 and $1.7 \mathrm{meV}$, depending on the number of $k$-points and the symmetry constraint (values in Table S3 of Supp. Inf.). We therefore conclude $1 \mathrm{meV}$ to be a typical error on the EOS energy.

The quality of the computed energies $E\left(V_{i}\right)$ is affected by noise from several phenomena, such as the incompleteness of the basis set, $k$-grid, or internal computational routines. To estimate the effect of this noise on the four EOS parameters $\left(V_{\min }, E_{\min }, B_{0}, B_{0}^{\prime}\right.$ in Eq. 3$)$, we apply uncorrelated random offsets to each of the energy data points $E\left(V_{i}\right)$, with the offsets uniformly distributed between $\pm 1 \mathrm{meV}$. The resulting changes in the four EOS parameters are then representative for the uncertainty caused by the noise. $10^{5}$ such datasets with random offsets are generated, and the standard deviation on each 
EOS parameter is taken as estimate for the error bar. These error bars vary between \pm 0.3 and $\pm 1.4 \AA^{3}$ for the equilibrium volume $V_{\min }$ and between \pm 0.1 and $\pm 0.2 \mathrm{GPa}$ for the bulk modulus $B_{0}$ depending on the number of $k$ points and the symmetry constraint (values in Table S3 of Supp. Inf.). Most differences in volume (see Table III) and bulk modulus (see Section IV C) caused by varying symmetry or $k$-point settings are therefore significant. Symmetry and $k$-point convergence appear to affect the equation of state in a systematic way, changing the shape of the curve as a whole rather than randomly distorting each data point. Indeed, $1 \mathrm{meV}$ error bars would be negligible on Fig. 3b. Our error analysis therefore shows that differences in $V_{\min }$ and $B_{0}$ values are not due to our fitting procedure, but entirely due to the numerical convergence phenomena that are the focus of this paper.

\section{Elastic constants and bulk modulus}

Table IV compares the computed stiffness tensor for the shape and EOS structures. The elasticity tensor $C$ has been directly obtained from the extended Hessian, using numerical differentiation of stresses and forces (Eq. 8). The lowest and highest eigenvalue of the stiffness tensor are listed, whose corresponding eigenvectors represent the easiest and most difficult deformation mode, respectively. ${ }^{37,57,58}$ The Voigt modulus $K_{V}$ and Reuss modulus $K_{R}$ are compared to the fitted bulk modulus $B_{0}$. All values are compared with those reported by Ortiz et al. ${ }^{37}$

We find the use of an EOS-optimized reference state to be crucial. The volume is not relaxed in the shape optimization, which leads to significantly different elastic constants. This means that the shape structure is already out of the linear elasticity regime; otherwise it would have identical elastic constants as the EOS structure. The EOS values, on the other hand, are in reasonable agreement with the values by Ortiz et al., despite the difference in functional and computational choices. The present work is based on the PBE functional with a $2 \times$ $2 \times 6 k$-point set, while the results of Ortiz et al. employ the B3LYP functional ${ }^{66}$ with a $3 \times 3 \times 3 k$-point set in CRYSTAL09.

More disturbing is the large difference between the Reuss modulus $K_{R}$ (Eqs. 8-10) and the fitted bulk modulus $B_{0}$ (Eq. 11) for the same EOS structure. Both $K_{R}$ and $B_{0}$ represent the response of the material to isotropic stress, and should in principle be identical. We posit three reasons for the discrepancy between $K_{R}$ and $B_{0}$.

First, $K_{R}$ is a linear response quantity, where the energy is assumed to depend quadratically on all degrees of freedom, i.e. both the ion positions and the unit cell dimensions. $K_{R}$ assumes the structure to be in the linear elasticity regime and assumes harmonic ion response. The $K_{R}$ value is thus the harmonic estimation, neglecting anharmonicities. In contrast, $B_{0}$ is based on the anharmonic Vinet expression $E_{\mathrm{EOS}}(V)$, where moreover the ion positions and unit cell shape are completely relaxed at every volume point. The $B_{0}$ value thus incorporates anharmonicities.

Second, the eggbox effect can introduce some noise in the elements of the Hessian. Errors in the Hessian propagate into errors in the relaxed-ion stiffness tensor $C$ because of Eq. 8, and subsequently in $K_{R}$ because of Eq. 10. The energy oscillates with an eggbox amplitude of approximately $0.1 \mathrm{meV}$ over a grid spacing of approximately $0.13 \AA$ (see Supporting Information). This gives errors on the Hessian elements of roughly $0.5 \mathrm{~kJ} / \mathrm{mol} / \AA^{2}$ per atom. Adding random uncorrelated uniform noise of $\pm 0.5 \mathrm{~kJ} / \mathrm{mol} / \AA^{2}$ to the Hessian elements gives, through Eq. 8, a small yet non-negligible standard deviation on the relaxed-ion stiffness tensor. For instance, the $C_{11}$ tensor element is affected by $\pm 0.46 \mathrm{GPa}$.

Third, the Pulay effect can affect the clamped-ion elastic constants. Errors in $C^{\text {clamp }}$ directly propagate into errors in $C$ because of Eq. 8. In our VASP calculations, the elastic constants are retrieved from a numerical differentiation of the stress tensor, which is calculated analytically for a number of deformed unit cells. These stress tensors are computed with a constant basis set. However, the desired stress tensor has a constant cutoff energy, and the difference is Pulay stress. The effect on the elastic constants is equal to the Pulay stress $P_{\text {Pulay }}$, as it can be regarded as evaluating the elastic constants of a material under stress. The error in $C$ elements is thus -0.125 GPa. Moreover, the used VASP routine restarts each calculation of a deformed unit cell from the wavefunction of the undeformed cell without updating the PW basis set size. Although this is much more efficient than re-initializing the wavefunction from scratch, it will change the apparent cutoff energy, which is another undesired basis set effect.

We illustrate these Pulay effects with the $C_{11}^{\text {clamp }}$ element, which is not affected by anharmonicities of the ions as the ions are not relaxed. The EOS reference structure is strained in the [100] direction by $\pm 1 \%$. In the energy-based approach, a parabola is fit through the three energies, and its curvature gives 98.4 GPa. This approach is free from any Pulay effect. Next, the stress tensor is constructed at every strained structure while re-initializing the wavefunction, in order to update the basis set between these strained structures. Its numerical differentiation gives a value of $98.3 \mathrm{GPa}$. This value is only affected by the Pulay stress, which indeed explains the difference of about 0.1 GPa. Finally, the stress tensor is constructed at every strained structure without re-initializing the wavefunction, to simulate the VASP routine. A value of $100.1 \mathrm{GPa}$ is found, which incorporates both Pulay stress and other basis set effects due to the change in apparent cutoff energy between the strained structures. In conclusion, the error in the $C_{11}^{\text {clamp }}$ element is of the order of $2 \mathrm{GPa}$.

Unfortunately, numerical inaccuracies in Hessian and stiffness tensor elements are enhanced due to numerical instabilities in the calculation of the Reuss modulus. $K_{R}$ 
TABLE IV. Elastic constants for MIL-47 (in GPa): relaxed-ion elastic tensor $C_{i j}$, minimum and maximum eigenvalue $c_{1}$ and $c_{6}$ of the $C$ tensor, Voigt modulus $K_{V}$, Reuss modulus $K_{R}$, and EOS bulk modulus $B_{0}$. The CRYSTAL values of Ortiz et al. are included for comparison ${ }^{37}$.

\begin{tabular}{r|rrrrrrrrrr|rrrrr}
\hline structures & $k$-points & $C_{11}$ & $C_{22}$ & $C_{33}$ & $C_{44}$ & $C_{55}$ & $C_{66}$ & $C_{12}$ & $C_{13}$ & $C_{23}$ & $c_{1}$ & $c_{6}$ & $K_{V}$ & $K_{R}$ & $B_{0}$ \\
\hline shape & $2 \times 2 \times 6$ & 76.8 & 27.6 & 35.0 & 40.4 & 6.1 & 7.8 & 44.2 & 15.0 & 8.5 & 1.6 & 107.2 & 30.5 & 19.4 & \\
EOS & $2 \times 2 \times 6$ & 67.6 & 34.0 & 35.4 & 44.2 & 6.7 & 8.7 & 46.0 & 15.2 & 10.2 & 1.8 & 104.7 & 31.1 & 13.6 & 6.1 \\
Ortiz $^{37}$ & $3 \times 3 \times 3$ & 62.6 & 36.2 & 40.7 & 50.8 & 7.8 & 9.3 & 47.0 & 12.6 & 9.3 & 0.9 & 96.6 & 30.8 & 9.7 & \\
\hline
\end{tabular}

involves both a pseudo-inverse of the Hessian to compute the relaxed-ion stiffness tensor $C$ (Eq. 8) as well as the inversion of $C$ to construct the compliance tensor $S$ (Eq. 10). For the particular case of MIL-47(V), the inversion of $C$ is numerically very sensitive. Indeed, the condition number (the ratio $c_{6} / c_{1}$ of the highest/lowest eigenvalues of $C$ ) amounts to fairly high values of 57 and 176 for our $2 \times 2 \times 6$ EOS structure and for the structure of Ortiz, respectively. The Pulay effects of the order of $2 \mathrm{GPa}$ then make all the difference: lowering only the $C_{11}$ element by $2 \mathrm{GPa}(3 \%)$, for example, yields a decrease in $K_{R}$ of $19 \%$. In comparison, it yields a decrease in $K_{V}$ of only $0.7 \%$, since the Voigt modulus does not require the inversion of $C$ and is therefore fairly insensitive to errors in $C$. The physical origin of the numerical instability of $K_{R}$ is specific to the flexible framework MIL-47(V): it is due to the strong coupling between the $a$ and $b$ directions.

Based on this analysis of the stiffness tensor, we conclude that numerical differentiation of stresses at deformed unit cells is not suitable to determine the compliance tensor or the Reuss modulus of breathing MOFs, even if it is a cheap and easily implementable methodology. It suffers from Pulay effects which are enhanced dramatically due to the coupling between the unit cell axes. Numerical differentiation of energies to construct $C$ can limit Pulay effects. When aiming for single crystal values, and as long as computational resources permit, we advise to construct a series of $E\left(V_{i}\right)$ points and to fit an equation of state, as we regard the fitted bulk modulus $B_{0}$ to be the least error prone, and in addition, $B_{0}$ takes care of anharmonicities of the ions.

\section{LARGE PORE VERSUS NARROW PORE OF MIL-47(V)}

\section{A. Structure comparison}

The MIL-47(V) MOF is a special case in the family of breathing MOFs. In contrast to other members of this family (e.g. MIL-53( $\mathrm{Al})$ ), it only shows breathing behavior under the application of a significant external pressure. ${ }^{23,26}$ Using the insights and methodology developed in the previous sections, the narrow-pore (NP) version of the MIL-47(V) will now be investigated (Fig. 1b). As initial geometry, we start from the structure provided in literature ${ }^{26}$ with lattice parameters presented in Table I (geom3), showing an almost 50\% reduction in unit cell volume. A $k$-point convergence test for this struc- ture yields results comparable to those observed for the large-pore MIL-47(V) (see Supporting Information). A $\Gamma$-centered $2 \times 6 \times 6 k$-point set corresponds to 40 irreducible $k$-points and offers a trade-off between accuracy and computational cost.

Using a fit to the Vinet equation of state (Eq. 3), the equilibrium volume is found, and calculation of the frequencies shows no imaginary frequencies to be present for this NP structure. Table V shows the optimized fitting parameters. A measure for the overall quality of the fit is given by the rmsd (Eq. 12). Using an uncorrelated uniform noise distribution of $\pm 1 \mathrm{meV}$, error bars are calculated on all fitting parameters from $10^{5}$ noisy data sets. The equilibrium energy $E_{\min }$ and volume $V_{\min }$ show only a very small error bar for both the LP and NP structure. The derivative of the bulk modulus $B_{0}^{\prime}$ is most error prone.

The equilibrium volume of the NP structure is $904.8 \AA^{3}$, about $42 \%$ smaller than that of the LP structure. This value is in fairly good agreement with the experimental NP volume of $947 \AA^{3}$ for MIL-47( $\left.\mathrm{V}^{\mathrm{IV}}\right)^{26}$ and $908 \AA^{3}$ for MIL-47( $\left.\mathrm{V}^{\mathrm{III}}\right)^{25}$. Energetically, i.e. without taking into account temperature effects, the NP structure is only $13.8 \mathrm{meV}$ less stable than the LP structure.

In Table $\mathrm{V}$, the fitted bulk modulus $B_{0}$ roughly halves when going from the LP to the NP form. This may appear strange at first, but is in line with the strongly negative value of the pressure derivative of the bulk modulus $\mathrm{B}_{0}^{\prime}$. The latter shows that increasing the pressure on the MIL-47(V) LP structure leads to a significant decrease in its bulk modulus, while decreasing the pressure increases the MIL-47(V)'s resistance to deformation. In case of the NP structure, $B_{0}^{\prime}$ is much weaker and shows an opposite sign. As such, the resistance to deformation of the NP structure will increase with increasing pressure, i.e., it will be harder to compress the NP structure further.

The structural differences between LP and NP are mainly in the overall unit cell volume and shape with limited internal reorganization. The $a$ and $b$ lattice vectors change significantly, leading to a reduction of the pore opening angle $\delta$ from $80^{\circ}$ to $39^{\circ}$. The obtained lattice parameters are in good agreement with the experimental values for NP MIL-47( $\left.\mathrm{V}^{\mathrm{III}}\right)$ and MIL-47( $\left.\mathrm{V}^{\mathrm{IV}}\right) .^{25,26} \mathrm{In}$ addition to the volume change, the symmetry reduces from an orthorhombic cell for the LP structure to a monoclinic cell for the NP structure. Our computed $\beta$ lattice angle of $112.4^{\circ}$ is in good agreement with the experimental values ${ }^{25,26}$ of $114^{\circ}$ for NP MIL- $47\left(\mathrm{~V}^{\mathrm{IV}}\right)$ and $104-115^{\circ}$ for NP MIL-47( $\left.\mathrm{V}^{\mathrm{III}}\right)$. 
TABLE V. Comparison of the LP $(2 \times 2 \times 6 k$-points $)$ and NP $(2 \times 6 \times 6 k$-points $)$ structures of MIL-47(V). (1) Different parameters of the Vinet EOS (Eq. 3) are tabulated with the error bars assuming a noise of $\pm 1 \mathrm{meV}$. The rmsd of the fit is also given (Eq. 12). (2) Structural parameters of the two structures: lattice parameters $a, b$, and $c$; lattice angle $\beta$ (the two other lattice angles $\alpha=\gamma=90.0^{\circ}$ ); opening angle $\delta$ of the pore; vanadium-oxide bond lengths $r_{\mathrm{VO}}^{\text {long }}$ and $r_{\mathrm{VO}}^{\text {short }}$ along the vanadyl chain; octahedral backbone angle $\tau$; superexchange angle $\sigma$; offset $b_{\mathrm{VV}}$ along the $b$-direction between neighboring $\mathrm{V}$ atoms in a vanadyl chain. (3) Elastic constants of the relaxed-ion stiffness tensor $C$, Voigt modulus $K_{V}$, Reuss modulus $K_{R}$. (4) Vibrational contributions at $300 \mathrm{~K}$ : zero-point energy (ZPE), vibrational internal energy $E_{\text {vib }}$, vibrational entropy ( $T S_{\text {vib }}$ ), vibrational Helmholtz free energy $F_{\mathrm{vib}}$, total Helmholtz free energy $F=\tilde{E}_{\mathrm{min}}+F_{\mathrm{vib}}$, and vibrational heat capacity $C_{V \text {,vib. }}$

(1) Vinet EOS fit parameters

\begin{tabular}{|c|c|c|c|c|c|}
\hline & $\tilde{E}_{\min }[\mathrm{meV}]$ & $V_{\min }\left[\AA^{3}\right]$ & $B_{0}[\mathrm{GPa}]$ & $B^{\prime}[-]$ & $\operatorname{rmsd}[\mathrm{meV}]$ \\
\hline LP & $-539932.6( \pm 0.3)$ & $1554.1( \pm 0.6)$ & $6.09 \quad( \pm 0.12)$ & $-55.0 \quad( \pm 2.1)$ & 0.472 \\
\hline NP & $-539918.8( \pm 0.3)$ & $904.8( \pm 1.4)$ & $2.84 \quad( \pm 0.18)$ & $15.9( \pm 10.1)$ & 0.046 \\
\hline LP-NP & $-13.8( \pm 0.6)$ & & & & \\
\hline
\end{tabular}

\begin{tabular}{|c|c|c|c|c|c|c|c|c|c|}
\hline & \multicolumn{9}{|c|}{ (2) Structural parameters } \\
\hline & $a$ & $b$ & $c$ & $\beta$ & $\delta$ & $r_{\mathrm{VO}}^{\text {long }}$ & $r_{\mathrm{VO}}^{\text {short }}$ & $\tau$ & $\sigma \quad b_{\mathrm{VV}}$ \\
\hline & {$[\AA]$} & {$[\AA]$} & {$[\AA]$} & {$\left[{ }^{\circ}\right]$} & {$\left[^{\circ}\right]$} & {$[\AA]$} & {$[\AA]$} & {$\left[{ }^{\circ}\right]$} & {$\left[{ }^{\circ}\right] \quad[\AA]$} \\
\hline LP & 16.394 & 13.854 & 6.842 & 90.19 & 80 & 2.08 & 1.66 & 175. & 133. 0.31 \\
\hline \multirow[t]{3}{*}{ NP } & 21.115 & 6.840 & 6.776 & 112.39 & 38 & 2.06 & 1.67 & 171. & 133. 0.43 \\
\hline & \multicolumn{9}{|c|}{ (3) Elastic constants and bulk modulus [in GPa] } \\
\hline & $C_{11}$ & $C_{22}$ & $C_{33}$ & $C_{44}$ & $C_{55}$ & $C_{66}$ & $C_{12}$ & $C_{13}$ & $\begin{array}{lll}C_{23} & K_{V} & K_{R}\end{array}$ \\
\hline $\mathrm{LP}$ & 67.6 & 34.0 & 35.4 & 44.2 & 6.7 & 8.7 & 46.0 & 15.2 & 10.231 .113 .6 \\
\hline NP & 198.6 & 6.4 & 41.4 & 7.3 & 1.7 & 20.6 & 12.3 & 37.6 & $\begin{array}{lll}3.5 & 39.2 \quad 5.7\end{array}$ \\
\hline
\end{tabular}

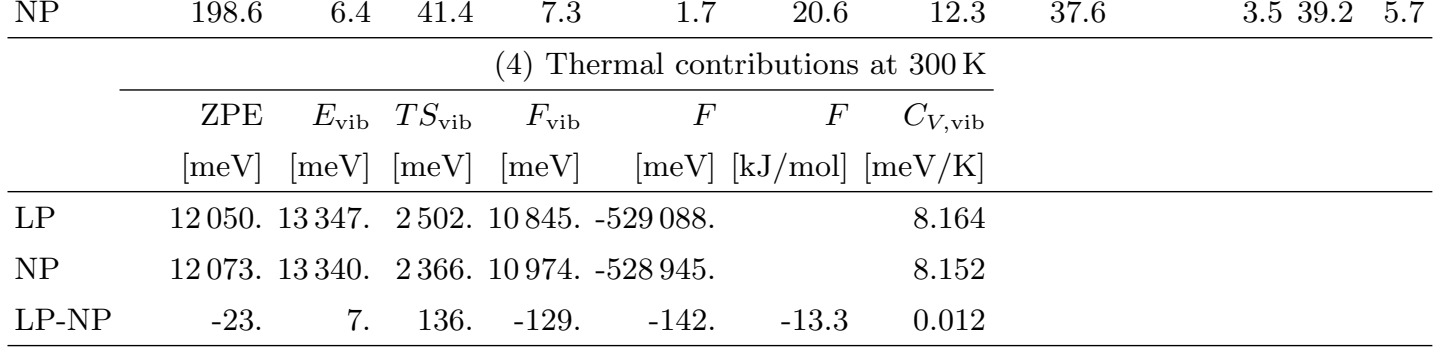

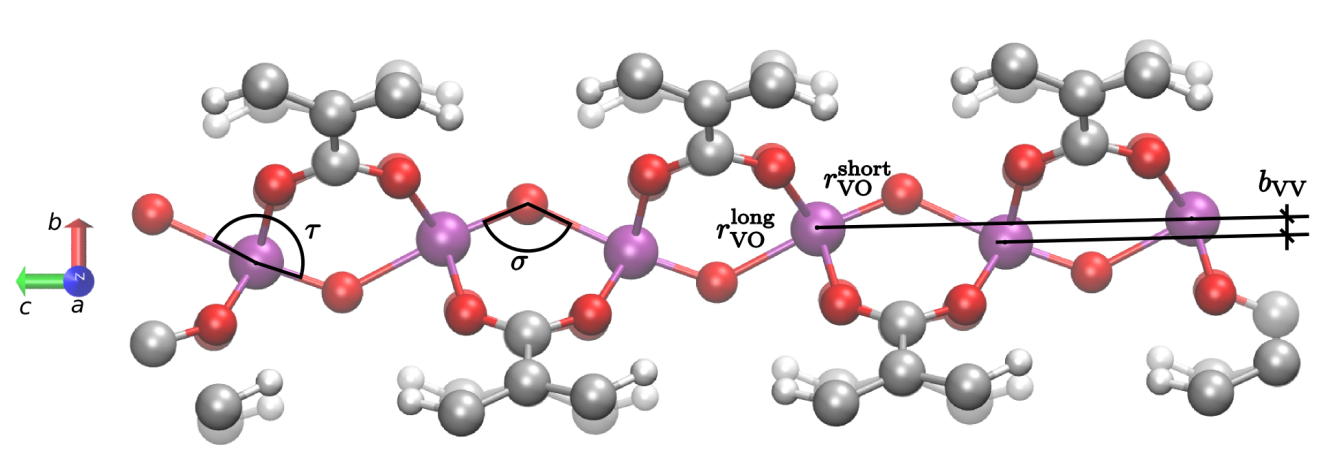

FIG. 5. Definition of structural parameters for Table V: vanadium-oxide distances $r_{\mathrm{VO}}^{\text {short }}$ and $r_{\mathrm{VO}}^{\text {long }}$ offset $b_{\mathrm{VV}}$, octahedral backbone angle $\tau$, and superexchange angle $\sigma$.

In both the LP and NP structure, the $\mathrm{VO}_{6}$ octahedra are asymmetrically distorted, as was already previously reported for the LP structure ${ }^{23,67}$. The structural parameters describing these distortions are defined in Fig. 5. The compressed apex $r_{\mathrm{VO}}^{\text {short }}$ of the octahedron is indicative of a double bond $(\mathrm{V}=\mathrm{O})$. The elongated apex $r_{\mathrm{VO}}^{\text {long }}$ is indicative for a trans bond $(\mathrm{V} \cdots \mathrm{O}) .{ }^{68}$ The octahedral backbone angle $\tau$ (angle $\mathrm{O}=\mathrm{V} \cdots \mathrm{O}$ ) is expected to be $180^{\circ}$ in a perfect octahedron, but it is distorted to $175^{\circ}$ for the LP structure and $171^{\circ}$ for the NP structure. In contrast, the superexchange angle $\sigma$ is the angle between subsequent octahedra in the vanadyl chain and re- 


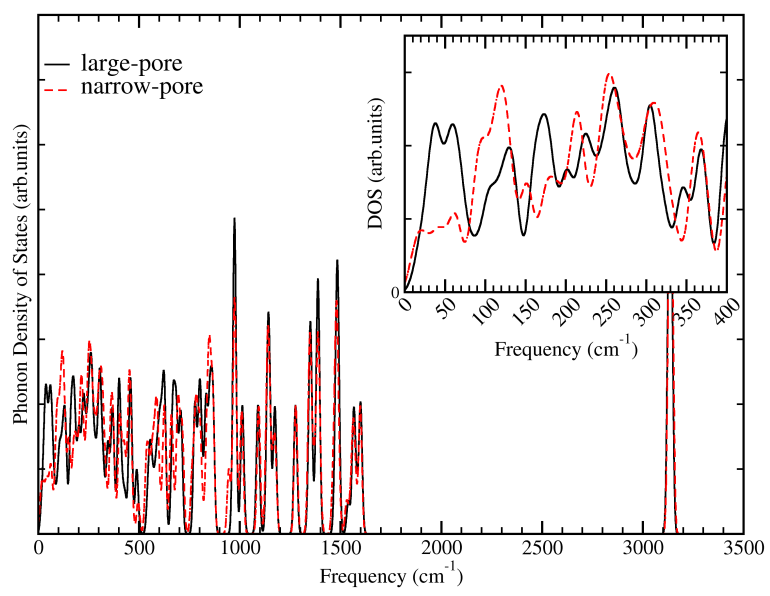

FIG. 6. Comparison of the phonon frequency spectrum of the LP (black curves) and the NP (red dashed curves) structures of MIL-47(V). A Gaussian smearing with a standard deviation of $8 \mathrm{~cm}^{-1}$ was applied to the computed frequencies. The inset zooms in on the low frequency region $\left(0-400 \mathrm{~cm}^{-1}\right)$.

mains identical between the LP and NP structures. The distortions in the $\tau$ angle and the compressed/elongated vanadium-oxygen distance $r_{\mathrm{VO}}^{\text {short }} r_{\mathrm{VO}}^{\text {long }}$ results in a zig-zag configuration of the vanadiums in a single vanadyl chain. The offset $b_{\mathrm{VV}}$ in the $b$-direction between neighboring vanadiums is 0.31 and $0.43 \AA$ for the LP and NP structure, respectively. Such a symmetry breaking in the $\mathrm{VO}_{6}$ octahedra has previously been shown to be energetically more favorable than a vanadyl chain of perfect octahedra. ${ }^{23}$

\section{B. Temperature effects}

Based on the computed phonon frequencies, a rough estimate of temperature effects can be made. This is very interesting for the system at hand, since finite temperature contributions to the (Helmholtz) free energy $F$ could shift the relative stability (of only $13.8 \mathrm{meV}$ at $0 \mathrm{~K}$ ) of the LP and NP structure. This is the case for other breathing MOFs; however, for the MIL-47(V) such thermally induced breathing has not been observed and is thus not expected.

At $0 \mathrm{~K}$, vibrational contributions to the free energy are limited to the zero-point energy (ZPE). As shown in Table V, the ZPE of the LP and NP structures differs by only $23 \mathrm{meV}$, and this difference stabilizes the LP structure further in comparison to the NP structure. At $300 \mathrm{~K}$, the vibrational entropic contribution $-T S_{\text {vib }}$ to the Helmholtz free energy favors the LP structure significantly (by $136 \mathrm{meV}$ ). The LP structure is indeed expected to have more freedom than the compact NP structure. A similar entropic stabilization of the LP was computed for other materials, for instance for the MIL-53(Ga) and MIL-53(Al) materials by Boutin et al. ${ }^{69}$ Overall, the to- tal Helmholtz free energy $F=\tilde{E}_{\text {min }}+F_{\text {vib }}$ of the LP structure is $142 \mathrm{meV}$ more stable than the NP structure at $300 \mathrm{~K}$. Thermal contributions thus stabilize the LP shape, which is in accordance with the LP MIL-47(V) structure being experimentally observed at finite temperature.

The phonon frequency spectrum in Fig. 6 is very similar for the LP and NP structure. Frequencies below $400 \mathrm{~cm}^{-1}$ show the largest differences (see inset of Fig. 6). The lowest non-zero frequency is as low as 18.6 and $13.1 \mathrm{~cm}^{-1}$ for the LP and NP, respectively. These and other low frequency motions describe twisting of the vanadyl chains with respect to each other and similar coherent large-scale motions involving a lot of mass.

Other particularly interesting frequencies are those that describe the linker flexibility. The terephthalate linkers are suspected to be able to rotate as hindered rotors. To investigate their behavior, the Partial Hessian Vibrational Analysis (PHVA) combined with the Mobile Block Hessian (MBH) approach is employed, i.e. the PHVA/MBH approach as proposed by Ghysels et al. ${ }^{70}$ In this combined approach, the terephthalate rings are treated as four mobile blocks that may translate and/or rotate without changing their internal geometry, while the other framework atoms are given an infinite mass such that they remain immobile in the vibrational analysis. This limits the degrees of freedom to only 24 frequencies, i.e. six per mobile block. The frequencies are identified to represent linker rotations by computing the variance in dihedral linker angles along the corresponding modes, which is also verified by visualization.

For the LP structure, these linker rotations (wagging) have the four lowest frequencies in the combined PHVA/MBH spectrum and are quasi-degenerate, corresponding to symmetric and asymmetric motions of the four linkers. The degeneracy implies that the individual benzene rotations are motions that couple little with each other. This is in line with the previously noted 1-D nature of this material, showing the vanadyl chains to be uncoupled. ${ }^{23}$ The linker rotations have a frequency of $42 \mathrm{~cm}^{-1}$ (energy $5.2 \mathrm{meV}$ ), which is in perfect agreement with the first peak at $5.2 \mathrm{meV}$ in inelastic neutron scattering experiments at $200 \mathrm{~K}{ }^{34}$

The benzene rotation frequencies $\omega$ may be linked to force constants by using the principal inertia moments $I$ of benzene. The force constant for the benzene rotation $\phi$ is estimated with $I \omega^{2}$ and is found to be approximately $1.3 \mathrm{~kJ} / \mathrm{mol} / \mathrm{rad}^{2}$. For the NP structure, the degeneracy of the linker rotations is broken, giving four different frequencies: 49.0 and $63.3 \mathrm{~cm}^{-1}$ for asymmetric rotations of pairs of benzenes, and the higher frequencies 116.8, and $125.5 \mathrm{~cm}^{-1}$ for symmetric rotations. This is seen in the inset of Fig. 6, which shows a suppression of the feature around $40-50 \mathrm{~cm}^{-1}$ in favor of a feature around $100 \mathrm{~cm}^{-1}$. The degeneracy is probably broken due to symmetry reduction of the unit cell to a monoclinic structure rather than an orthorhombic structure, in addition to symmetry breaking in the linker orientations of the NP structure. 
All four frequencies are higher for the NP than for the LP structure, indicating that linker mobility is reduced in the NP structure because of steric hindrance.

\section{CONCLUSION}

Modeling of flexible materials requires the computation of mechanical properties from first principles, e.g. for screening of materials in a database, for gaining insight in structural transformations, and for force field development. However, this paper shows that computations with periodic density functional theory are challenged by the flexibility of these materials: guidelines from experience with standard solid state calculations cannot be simply transferred to flexible porous frameworks.

The MIL-47(V) material has a large-pore and a narrow-pore shape and is used to illustrate the effect of $k$-point sampling in reciprocal space, symmetry effects, and the effect of Pulay stress. A $k$-point convergence test should preceed any computational study. The $k$ point set should be sufficiently dense in reciprocal space in each unit cell axis direction. An energy convergence of $1 \mathrm{meV}$ was reached for the MIL-47(V) material with the $2 \times 2 \times 6 k$-point set for the large-pore and $2 \times 6 \times 6$ $k$-point set for the narrow-pore structure.

Symmetry breaking had to be imposed by manually distorting the structure along its imaginary frequency eigenmodes. MIL-47(V) could successfully lower its energy by distorting the vanadium-oxide distances and angles in the vanadyl chains and by rotating the benzene linkers.

Pulay stress is an artificial stress that tends to lower the volume and is caused by the finite size of the plane wave basis set. During structure optimization, Pulay stress made MIL-47(V) leave its large-pore stable shape to collapse into the narrow-pore shape. As an improved optimization scheme, we propose to optimize subsequently the ion positions and the unit cell shape in a first step. In the next step, an energy-versus-volume profile $E(V)$ should be constructed, where the energies are computed by fixing the volume at discrete points and optimizing the ions and shape at each chosen volume. An equation of state model can then be fit through the computed energies. Finally, based on this EOS fit, the minimum energy and corresponding volume may be predicted. The volume prediction in this procedure is not affected by Pulay stress.

For the mechanical properties, the bulk modulus was computed in VASP using the harmonic approximation. It is based on numerical differentiation of the ion forces and the stress tensor. The clamped-ion stiffness tensor is corrected with an extra term for the response of the ion positions, to obtain the relaxed-ion stiffness tensor $C$. The Reuss modulus follows from taking the inverse of $C$. It describes the resistance of a single crystal to isotropic pressure, and it should in principle be identical to the volume derivative of the pressure, i.e. the curva- ture of the $\operatorname{EOS} E(V)$ curve. The harmonic approach can however differ from the EOS approach for three reasons: anharmonicity, eggbox effect, and Pulay effects. These errors propagate into the Reuss modulus. Indeed, the $a$ and $b$ axis of MIL-47(V) are strongly coupled when the unit cell deforms, which expresses itself in numerical instability in the inversion of $C$. The computed MIL-47(V) Reuss modulus is extremely sensitive to these effects. For a flexible material, it is therefore advisible to resort to the EOS fit procedure.

Lastly, the EOS structure optimization procedure has been repeated for the NP structure of MIL-47(V), and mechanical and thermal properties of the LP and NP structure have been compared. The predicted volumes lie fairly close to the experimental values. Energetically, the entropic contribution to the free energy favors the LP phase by $136 \mathrm{meV}$ at $300 \mathrm{~K}$, and overall the LP is computed to be more stable than the NP by $142 \mathrm{meV}$ at $300 \mathrm{~K}$. Symmetry breaking is again necessary to find a stable equilibrium point: the unit cell is monoclinic, degeneracy in linker rotation frequencies is removed, and vanadium-oxide distances are not all equivalent.

In summary, anisotropic flexibility makes a structure optimization of MOF materials a delicate task. We expect that equal caution should be taken for other materials with strong coupling between the lattice vectors, as is here the case for the $a$ and $b$ direction of MIL-47(V). Symmetry breaking should be allowed in the optimization, and the EOS procedure should be used to avoid the effects of Pulay stress inherent to a finite basis set. Further research may include the accurate characterization of the (free) energy barrier, which is essential in forcefield development.

\section{SUPPORTING INFORMATION}

The supporting information includes 1) visualization of eggbox effect for the large-pore (LP) structure, 2) $k$ point convergence of the energy for the narrow-pore (NP) structure, similarly to Table II, 3) bulk moduli computed for LP structures with various computational settings, 4) comparison of Vinet EOS to Birch-Murnaghan EOS, 5) parameters obtained from EOS fit, and 6) Pulay stress tensor with various computational settings, 7) Hirshfeld-I charges. The LP and NP structure from the EOS optimization procedure with $2 \times 2 \times 6$ and $2 \times 6 \times 6 k$-points respectively are available in the database of the Cambridge Crystallographic Data Centre (CCDC 1419980 for LP, CCDC 1419981 for NP).

\section{ACKNOWLEDGMENTS}

The computational resources and services used in this work were provided by Ghent University (Stevin), the Hercules Foundation (Tier-1 Flemish Supercomputer Infrastructure), and the Flemish Government - Depart- 
ment of EWI. Funding was received from the Research Board of Ghent University (BOF) and the Research Foundation Flanders (FWO). D.E.P. is a postdoctoral researcher funded by the FWO (project number $12 \mathrm{~S} 3415 \mathrm{~N})$. V.V.S. acknowledges funding from the Eu- ropean Research Council under the European Commission's Seventh Framework Programme (FP7(2007-2013) ERC grant agreement number 240483). We thank Wim De Witte for help with the figures and Louis Vanduyfhuys and Toon Verstraelen for fruitful discussions.
1 Long, J. R.; Yaghi, O. M. the Pervasive Chemistry of Metal-Organic Frameworks. Chem. Soc. Rev. 2009, 38, 1213-1214.

2 Zhang, S.-Y.; Zhang, Z.; Zaworotko, M. J. Topology, Chirality and Interpenetration in Coordination Polymers. Chem. Commun. 2013, 49, 9700-9703.

3 Rosseinsky, M. Recent Developments in Metal-Organic Framework Chemistry: Design, Discovery, Permanent Porosity and Flexibility. Micropor. Mesopor. Mat. 2004, 73, 15-30.

4 Alaerts, L.; Kirschhock, C. E. A.; Maes, M.; van der Veen, M. A.; Finsy, V.; Depla, A.; Martens, J. A.; Baron, G. V.; Jacobs, P. A.; Denayer, J. E. M.; De Vos, D. E. Selective Adsorption and Separation of Xylene Isomers and Ethylbenzene with the Microporous Vanadium(IV) Terephthalate MIL-47. Angew. Chem. Int. Ed. 2007, 46, 4293-4297.

${ }^{5}$ Rosenbach, N.; Jobic, H.; Ghoufi, A.; Salles, F.; Maurin, G.; Bourrelly, S.; Llewellyn, P. L.; Devic, T.; Serre, C.; Férey, G. Quasi-Elastic Neutron Scattering and Molecular Dynamics Study of Methane Diffusion in Metal Organic Frameworks MIL-47(V) and MIL-53(Cr). Angew. Chem. (Int. Ed.) 2008, 47, 6611-5.

6 Murray, L. J.; Dinca, M.; Long, J. R. Hydrogen Storage in Metal-Organic Frameworks. Chem. Soc. Rev. 2009, 38, 1294-1314.

7 Bueno-Pérez, R.; Garcia-Perez, E.; Jose GutierrezSevillano, J.; Merkling, P. J.; Calero, S. A Simulation Study of Hydrogen in Metal-Organic Frameworks. Adsorpt. Sci. Technol. 2010, 28, 823-835, 35th Iberian Meeting on Adsorption, Univ Lisbon, Fac Sci, Lisbon, Portugal, Sep 08-10, 2010.

${ }^{8}$ Gascon, J.; Kapteijn, F. Metal-Organic Framework Membranes-High Potential, Bright Future? Angew. Chem. Int. Ed. 2010, 49, 1530-1532.

9 Biswas, S.; Vanpoucke, D. E. P.; Verstraelen, T.; Vandichel, M.; Couck, S.; Leus, K.; Liu, Y.-Y.; Waroquier, M.; Van Speybroeck, V.; Denayer, J. F. M.; Van Der Voort, P. New Functionalized Metal-Organic Frameworks MIL-47-X (X = - Cl, - Br, $\left.-\mathrm{CH}_{3},-\mathrm{CF}_{3},-\mathrm{OH},-\mathrm{OCH}_{3}\right)$ : Synthesis, Characterization, and $\mathrm{CO}_{2}$ Adsorption Properties. J. Phys. Chem. C 2013, 117, 22784-22796.

10 Llewellyn, P. L.; Bourrelly, S.; Vagner, C.; Heymans, N.; Leclerc, H.; Ghoufi, A.; Bazin, P.; Vimont, A.; Daturi, M.; Devic, T.; Serre, C.; Weireld, G. D.; Maurin, G. Evaluation of MIL-47(V) for $\mathrm{CO}_{2}$-Related Applications. J. Phys. Chem. C 2013, 117, 962-970.

11 Ramsahye, N. A.; Maurin, G.; Bourrelly, S.; Llewellyn, P. L.; Devic, T.; Serre, C.; Loiseau, T.; Férey, G. Adsorption of $\mathrm{CO} 2$ in Metal Organic Frameworks of Different Metal Centres: Grand Canonical Monte Carlo Simulations Compared to Experiments. Adsorption 2007, 13, 461-467.
12 Yazaydın, A. O.; Snurr, R. Q.; Park, T.-H.; Koh, K.; Liu, J.; LeVan, M. D.; Benin, A. I.; Jakubczak, P.; Lanuza, M.; Galloway, D. B.; Low, J. J.; Willis, R. R. Screening of Metal-Organic Frameworks for Carbon Dioxide Capture from Flue Gas Using a Combined Experimental and Modeling Approach. JACS 2009, 131, 1819818199.

13 Kolokolov, D. I.; Jobic, H.; Stepanov, A. G.; Ollivier, J.; Rives, S.; Maurin, G.; Devic, T.; Serre, C.; Férey, G. Experimental and Simulation Evidence of a Corkscrew Motion for Benzene in the Metal-Organic Framework MIL-47. J. Phys. Chem. C 2012, 116, 15093-15098.

14 Ghysels, A.; Vanduyfhuys, L.; Vandichel, M.; Waroquier, M.; Van Speybroeck, V.; Smit, B. on the Thermodynamics of Framework Breathing: A Free Energy Model for Gas Adsorption in MIL-53. J. Phys. Chem. C 2013, $117,11540-11554$.

15 Vanduyfhuys, L.; Verstraelen, T.; Vandichel, M.; Waroquier, M.; Van Speybroeck, V. Ab Initio Parametrized Force Field for the Flexible Metal-Organic Framework MIL-53(Al). J. Chem. Theory Comput. 2012, 8, 32173231.

16 Stroppa, A.; Jain, P.; Barone, P.; Marsman, M.; PerezMato, J. M.; Cheetham, A. K.; Kroto, H. W.; Picozzi, S. Electric Control of Magnetization and Interplay Between Orbital Ordering and Ferroelectricity in a Multiferroic MetalOrganic Framework. Angew. Chem. Int. Ed. 2011, 50, 5847-5850.

17 Combelles, C.; Yahia, M. B.; Pedesseau, L.; Doublet, M.L. $\mathrm{Fe}^{I I} / \mathrm{Fe}^{I I I}$ Mixed-Valence State Induced by Li-Insertion into the Metal-Organic-Framework Mil53(Fe): A DFT+U Study. J. Power Sources 2011, 196, 3426 - 3432.

18 Sillar, K.; Sauer, J. Ab Initio Prediction of Adsorption Isotherms for Small Molecules in Metal-Organic Frameworks: The Effect of Lateral Interactions for Methane/CPO-27-Mg. J. Am. Chem. Soc. 2012, 134, 18354-18365.

19 Flage-Larsen, E.; Røyset, A.; Cavka, J. H.; Thorshaug, K. Band Gap Modulations in UiO Metal-Organic Frameworks. J. Phys. Chem. C 2013, 117, 20610-20616.

20 Wang, Z.; Jain, P.; Choi, K.-Y.; van Tol, J.; Cheetham, A. K.; Kroto, H. W.; Koo, H.-J.; Zhou, H.; Hwang, J.; Choi, E. S.; Whangbo, M.-H.; Dalal, N. S. Dimethylammonium Copper Formate $\left[\left(\mathrm{CH}_{3}\right)_{2} \mathrm{NH}_{2}\right] \mathrm{Cu}(\mathrm{HCOO})_{3}$ : A Metal-Organic Framework with Quasi-One-Dimensional Antiferromagnetism and Magnetostriction. Phys. Rev. B 2013, 87, 224406.

21 Chen, X.; Wang, Y.-Y.; Liu, B.; Yin, B.; Liu, P.; Shi, Q.-Z. New Two-Dimensional Mn(II) Metal-Organic Framework Featured Spin Canting. Dalton Trans. 2013, 42, 70927100.

${ }^{22}$ Flage-Larsen, E.; Thorshaug, K. Linker Conformation Effects on the Band Gap in Metal-Organic Frameworks. Inorganic Chemistry 2014, 53, 2569-2572. 
${ }^{23}$ Vanpoucke, D. E. P.; Jaeken, J. W.; Baerdemacker, S. D.; Lejaeghere, K.; Speybroeck, V. V. Quasi-1D Physics in Metal-Organic Frameworks: MIL-47(V) from First Principles. Beilstein J. Nanotechnol. 2014, 5, 1738-1748.

24 Serre, C.; Bourrelly, S.; Vimont, A.; Ramsahye, N. A.; Maurin, G.; Llewellyn, P. L.; Daturi, M.; Filinchuk, Y.; Leynaud, O.; Barnes, P.; Férey, G. an Explanation for the Very Large Breathing Effect of a Metal-Organic Framework During $\mathrm{CO}_{2}$ Adsorption. Adv. Mater. 2007, 19, 2246-2251.

${ }^{25}$ Leclerc, H.; Devic, T.; Devautour-Vinot, S.; Bazin, P.; Audebrand, N.; Férey, G.; Daturi, M.; Vimont, A.; Clet, G. Influence of the Oxidation State of the Metal Center on the Flexibility and Adsorption Properties of a Porous Metal Organic Framework: MIL-47(V). J. Phys. Chem. C 2011, 115, 19828-19840.

26 Yot, P. G.; Ma, Q.; Haines, J.; Yang, Q.; Ghoufi, A.; Devic, T.; Serre, C.; Dmitriev, V.; Ferey, G.; Zhong, C.; Maurin, G. Large Breathing of the MOF MIL-47 $\left(\mathrm{V}^{\mathrm{IV}}\right)$ Under Mechanical Pressure: A Joint Experimental-Modelling Exploration. Chem. Sci. 2012, 3, 1100-1104.

27 Alhamami, M.; Doan, H.; Cheng, C.-H. A Review on Breathing Behaviors of Metal-Organic-Frameworks (MOFs) for Gas Adsorption. Materials 2014, 7, 31983250 .

${ }^{28}$ Lejaeghere, K.; Van Speybroeck, V.; Van Oost, G.; Cottenier, S. Error Estimates for Solid-State DensityFunctional Theory Predictions: An Overview by Means of the Ground-State Elemental Crystals. Critical Reviews in Solid State and Materials Sciences 2014, 39, 1-24.

29 Tan, J. C.; Civalleri, B.; Lin, C. C.; Valenzano, L.; Galvelis, R.; Chen, P. F.; Bennett, T. D.; MellotDraznieks, C.; Zicovich-Wilson, C. M.; Cheetham, A. K. Exceptionally Low Shear Modulus in a Prototypical Imidazole-Based Metal-Organic Framework Material. Phys. Rev. Lett. 2012, 108, 095502.

30 Walker, A. M.; Civalleri, B.; Slater, B.; MellotDraznieks, C.; Corà, F.; Zicovich-Wilson, C. M.; RománPérez, G.; Soler, J. M.; Gale, J. D. Flexibility in a MetalOrganic Framework Material Controlled by Weak Dispersion Forces: The Bistability of MIL-53(Al). Angew. Chem. Int. Ed. 2010, 49, 7501-7503.

31 Coudert, F. X.; Mellot-Draznieks, C.; Fuchs, A. H.; Boutin, A. Double Structural Transition in Hybrid Material MIL-53 upon Hydrocarbon Adsorption: The Thermodynamics Behind the Scenes. J. Am. Chem. Soc. 2009, 131, 3442-3443.

32 Coudert, F. X.; Mellot-Draznieks, C.; Fuchs, A. H.; Boutin, A. Prediction of Breathing and Gate-Opening Transitions upon Binary Mixture Adsorption in MetalOrganic Frameworks. J. Am. Chem. Soc. 2009, 131, 11329-11332.

33 Neimark, A. V.; Coudert, F.; Boutin, A.; Fuchs, A. H. Stress-Based Model for the Breathing of Metal-Organic Frameworks. J. Phys. Chem. Lett. 2010, 1, 445-449.

${ }^{34}$ Kolokolov, D.; Jobic, H.; Stepanov, A.; Plazanet, M.; Zbiri, M.; Ollivier, J.; Guillerm, V.; Devic, T.; Serre, C.; Férey, G. Comparison of the Dynamics of MIL-53(Cr) and MIL-47(V) Frameworks Using Neutron Scattering and DFT Methods. Eur. Phys. J. Special Topics 2010, 189, 263-271.

${ }^{35}$ Hendon, C. H.; Tiana, D.; Fontecave, M.; Sanchez, C.; D'arras, L.; Sassoye, C.; Rozes, L.; Mellot-Draznieks, C.; Walsh, A. Engineering the Optical Response of the
Titanium-MIL-125 Metal-Organic Framework Through Ligand Functionalization. JACS 2013, 135, 10942-10945.

${ }^{36}$ Liu, Y.; Her, J. H.; Dailly, A.; Ramirez-Cuesta, A. J.; Neumann, D.; Brown, C. M. Reversible Structural Transition in MIL-53 with Large Temperature Hysteresis. J. Am. Chem. Soc. 2008, 130, 11813-11818.

37 Ortiz, A. U.; Boutin, A.; Fuchs, A. H.; Coudert, F. Anisotropic Elastic Properties of Flexible Metal-Organic Frameworks: How Soft Are Soft Porous Crystals? Phys. Rev. Lett. 2012, 109, 195502.

38 Piccini, G.; Sauer, J. Quantum Chemical Free Energies: Structure Optimization and Vibrational Frequencies in Normal Modes. J. Chem. Theor. Comput. 2013, 9, 50385045 .

39 Barthelet, K.; Marrot, J.; Riou, D.; Férey, G. A Breathing Hybrid Organic - Inorganic Solid with Very Large Pores and High Magnetic Characteristics. Angew. Chem. Int. Ed. 2002, 41, 281.

40 Wang, X.; Liu, L.; Jacobson, A. J. Intercalation of Organic Molecules into Vanadium(IV) Benzenedicarboxylate: Adsorbate Structure and Selective Absorption of Organosulfur Compounds. Angew. Chem. Int. Ed. 2006, 45, 64996503.

41 Wang, X.; Eckert, J.; Liu, L.; Jacobson, A. J. Breathing and Twisting: An Investigation of Framework Deformation and Guest Packing in Single Crystals of a Microporous Vanadium Benzenedicarboxylate. Inorg. Chem. 2011, 50, 2028-2036.

42 Monkhorst, H. J.; Pack, J. D. Special Points for BrillouinZone Integrations. Phys. Rev. B 1976, 13, 5188-5192.

43 Froyen, S.; Cohen, M. L. Structural Properties of $\mathrm{NaCl}$ and KCl Under Pressure. J. Phys. C: Solid State Phys. 1986, 19, 2623-2632.

44 Gomes Dacosta, P.; Nielsen, O. H.; Kunc, K. Stress Theorem in the Determination of Static Equilibrium by the Density Functional Method. J. Phys. C: Solid State Phys. 1986, 19, 3163-3172.

45 Vanderbilt, D. Absence of Large Compressive Stress on Si(111). Phys. Rev. Lett. 1987, 59, 1456-1459.

46 Francis, G. P.; Payne, M. C. Finite Basis Set Corrections to Total Energy Pseudopotential Calculations. J. Phys.: Condens. Matter 1990, 2, 4395-4404.

47 Birch, F. Finite Elastic Strain of Cubic Crystals. Physical Review 1947, 71, 809-824.

48 Vinet, P.; Ferrante, J.; Rose, J. H.; Smith, J. R. Compressibility of Solids. Journal of Geophysical Research 1987, 92, 9319-9325.

49 Hama, J.; Suito, K. The Search for a Universal Equation of State Correct up to Very High Pressures. Journal of Physics: Condensed Matter 1996, 8, 67-81.

50 Rose, J. H.; Ferrante, J.; Smith, J. R. Universal Binding Energy Curves for Metals and Bimetallic Interfaces. Physical Review Letters 1981, 47, 675-678.

51 Cui, Q.; Bahar, I. Normal Mode Analysis: Theory and Applications to Biological and Chemical Systems; Chapman and Hall/CRC, 2006.

52 McQuarrie, D. A.; Simon, J. D. Physical Chemistry: A Molecular Approach; University Science Books: 55D Gate Five Road, Sausalito, CA 94965, 1997.

${ }^{53}$ Wu, X.; Vanderbilt, D.; Hamann, D. R. Systematic Treatment of Displacements, Strains, and Electric Fields in Density-Functional Perturbation Theory. Phys. Rev. B 2005, 72, 035105 . 
${ }^{54}$ Woodcock, H. L.; Zheng, W. J.; Ghysels, A.; Shao, Y. H.; Kong, J.; Brooks, B. R. Vibrational Subsystem Analysis: A Method for Probing Free Energies and Correlations in the Harmonic Limit. J. Chem. Phys. 2008, 129, 214109.

55 Ghysels, A.; Van Speybroeck, V.; Pauwels, E.; Catak, S.; Brooks, B. R.; Van Neck, D.; Waroquier, M. Comparative Study of Various Normal Mode Analysis Techniques Based on Partial Hessians. J. Comput. Chem. 2010, 31, 9941007.

56 Ghysels, A.; Miller, B. T.; Pickard, F. C.; Brooks, B. R. Comparing Normal Modes Across Different Models and Scales: Hessian Reduction Versus Coarse-Graining. J. Comput. Chem. 2012, 33, 2250-2275.

57 Ortiz, A. U.; Boutin, A.; Fuchs, A. H.; Coudert, F. X. Metalorganic Frameworks with Wine-Rack Motif: What Determines Their Flexibility and Elastic Properties? J. Chem. Phys. 2013, 138, 174703.

58 Marmier, A.; Lethbridge, Z. A. D.; Walton, R. I.; Smith, C. W.; Parker, S. C.; Evans, K. E. ElAM: a Computer Program for the Analysis and Representation of Anisotropic Elastic Properties. Comput. Phys. Commun. 2010, 181, 2102-2115.

59 Allen, F. H. The Cambridge Structural Database: A Quarter of a Million Crystal Structures and Rising. Acta Crystall. 2002, B58, 380-388.

${ }^{60}$ Perdew, J. P.; Burke, K.; Ernzerhof, M. Generalized Gradient Approximation Made Simple. Phys. Rev. Lett. 1996, 77, 3865-3868.

61 Csonka, G.; Perdew, J.; Ruzsinszky, A.; Philipsen, P.; Lebègue, S.; Paier, J.; Vydrov, O.; Ângyán, J. Assessing the Performance of Recent Density Functionals for Bulk Solids. Phys. Rev. B 2009, 79, 155107.

62 Haigis, V.; Belkhodja, Y.; Franois-Xavier Coudert, F.; Vuilleumier, R.; Anne Boutin, A. Challenges in FirstPrinciples NPT Molecular Dynamics of Soft Porous Crystals: A Case Study on MIL-53(Ga). J. Chem. Phys. 2014, $141,064703$.

${ }^{63}$ Grimme, S.; Antony, J.; Ehrlich, S.; Krieg, H. A Consistent and Accurate Ab Initio Parametrization of Density Functional Dispersion Correction (DFT-D) for the 94 Elements H-Pu. J. Chem. Phys. 2010, 132, 154104.

${ }^{64}$ Grimme, S.; Ehrlich, S.; Goerigk, L. Effect of the Damping Function in Dispersion Corrected Density Functional Theory. J. Comput. Chem. 2011, 32, 1456-1465.

65 Vanduyfhuys, L.; Ghysels, A.; Rogge, S.; Demuynck, R.; Van Speybroeck, V. Semi-Analytical Mean-Field Model for Predicting Breathing in MetalOrganic Frameworks. Molecular Simulation 2015 , DOI:10.1080/08927022.2015.1048512.

66 Becke, A. D. Density-Functional Thermochemistry. III. The Role of Exact Exchange. J. Chem. Phys. 1993, 98, 5648 .

67 Meilikhov, M.; Yusenko, K.; Torrisi, A.; Jee, B.; MellotDraznieks, C.; Pöppl, A.; Fischer, R. A. Reduction of a Metal-Organic Framework by an Organometallic Complex: Magnetic Properties and Structure of the Inclusion Compound [(?5-C5H5)2Co]0.5@MIL-47(V). Angew. Chem. Int. Ed. 2010, 49, 6212-6215.

${ }^{68}$ Weeks, C.; Song, Y.; Suzuki, M.; Chernova, N. A.; Zavalij, P. Y.; Whittingham, M. S. The One Dimensional Chain Structures of Vanadyl Glycolate and Vanadyl Acetate. J. Mater. Chem. 2003, 13, 1420-1423.

69 Coudert, F. X.; Ortiz, A. U.; Haigis, V.; Bousquet, D.; Fuchs, A. H.; Ballandras, A.; Weber, G.; Bezverkhyy, I.;
Geoffroy, N.; Bellat, J.; Ortiz, G.; Chaplais, J.; Patarin, J.; Boutin, A. Water Adsorption in Flexible Gallium-Based MIL-53 MetalOrganic Framework. J. Phys. Chem. C 2014, 118, 53975405 .

70 De Moor, B. A.; Ghysels, A.; Reyniers, M. F.; Van Speybroeck, V.; Waroquier, M.; Marin, G. B. Normal Mode Analysis in Zeolites: Toward an Efficient Calculation of Adsorption Entropies. J. Chem. Theory Comput. 2011, 7, 1090-1101. 


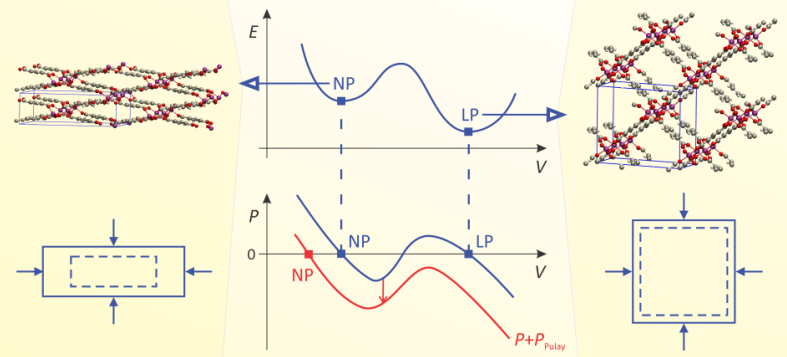

FIG. 7. Figure for table of contents. 